\title{
An Orthotropic Mesh Corrected Crack Model
}

M. Cervera 


\title{
An Orthotropic Mesh Corrected Crack Model
}

\author{
M. Cervera
}

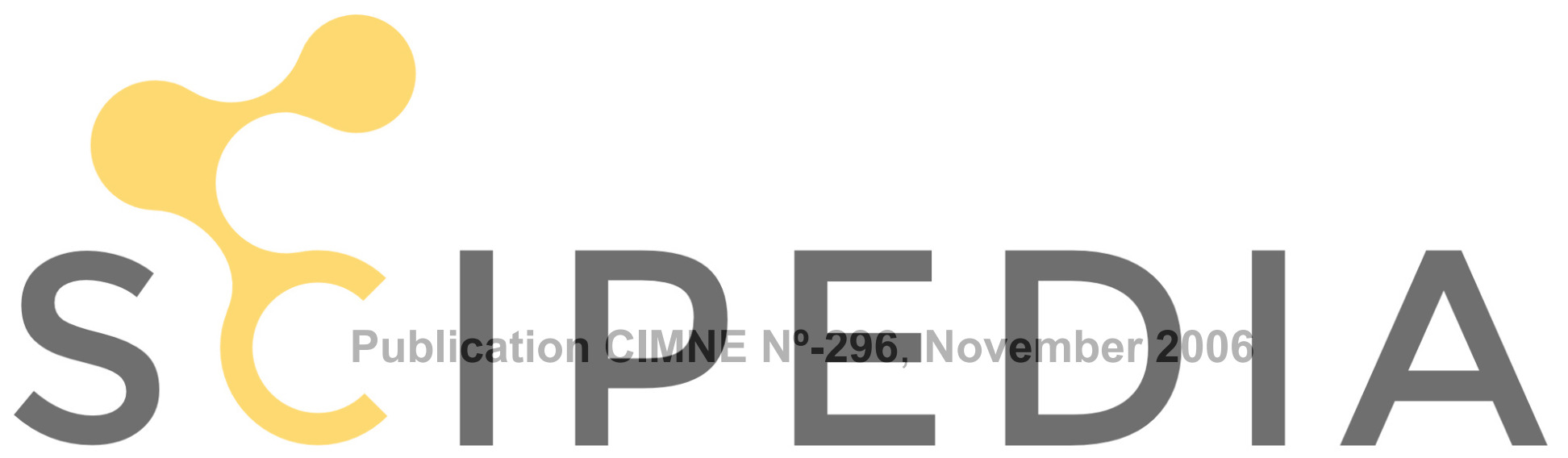

Register for free at https//www.scipedia.com to download the version without the watermark 


\title{
An Orthotropic Mesh Corrected Crack Model
}

\author{
M. Cervera \\ International Center for Numerical Methods in Engineering (CIMNE) \\ Technical University of Catalonia (UPC) \\ Edificio C1, Campus Norte, Jordi Girona 1-3, 08034 Barcelona, Spain.
}

KEYWORDS: tensile cracking, orthotronic damage, strain softening, non-associativity,

shear locking, smeared-embedded models.

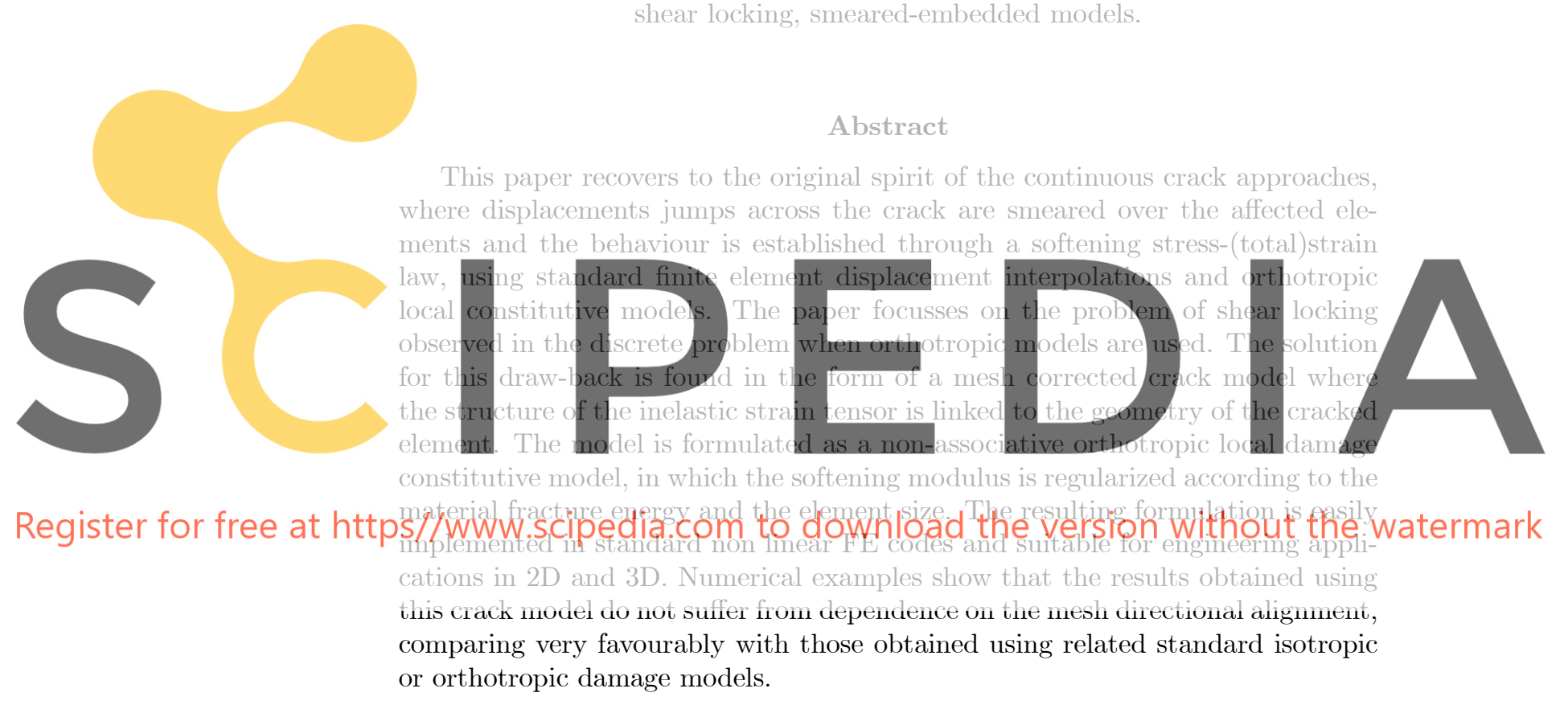




\section{Introduction}

Tensile cracking is an essential feature of the behaviour of concrete structures and, therefore, tensile fracture must be taken into account in predicting their ultimate load capacity as well as service behavior.

With the advent of digital computers and computational mechanics, two different formats have evolved to model the phenomenon of tensile cracking in the context of finite element analysis: the discontinuous and the continuous crack approaches. In the discontinuous crack models, displacements jumps across the crack are explicitly considered and the behaviour is established through a softening traction-jump law. In the continuous crack models, displacements jumps across the crack are smeared over the affected elements and the behaviour of the crack is established through a softening

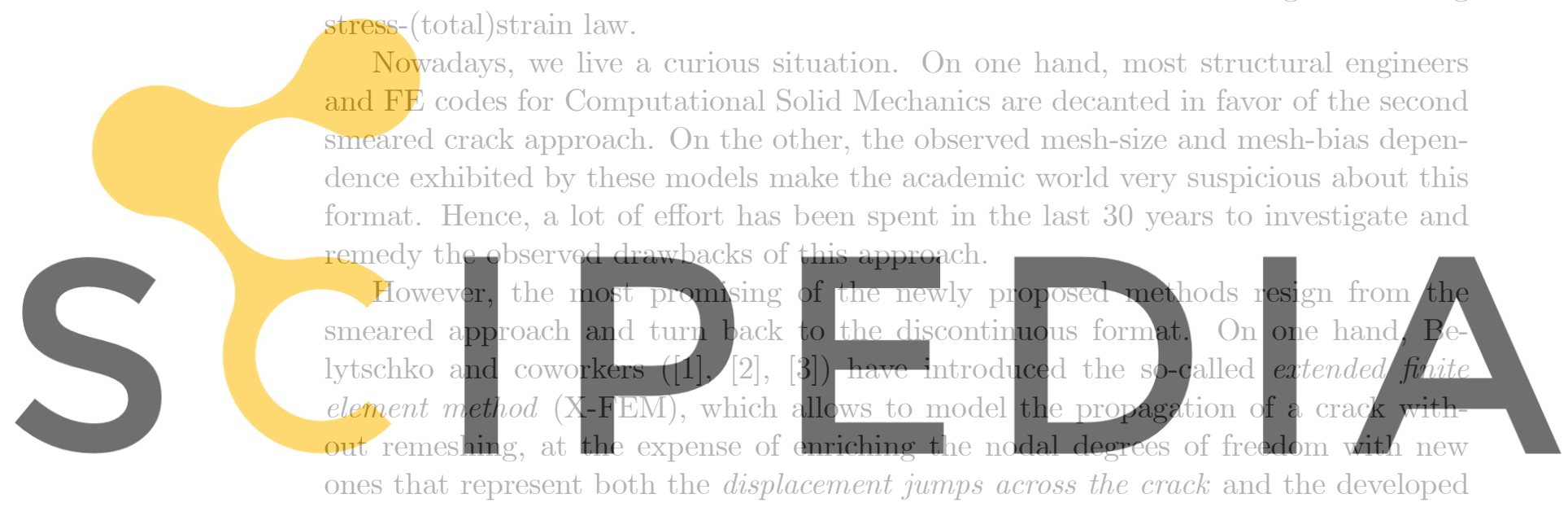

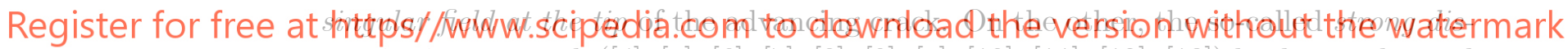
continuity approach ([4], [5], [6], [7], [8], [9], [5], [10], [11], [12], [13]) leads to enhanced formulations for finite elements with embedded displacement discontinuities.

But there is still one option to investigate: the adoption of smeared models which incorporate the effect of the displacement jumps in the strain field of the elements, rather than the actual jumps themselves. These could be termed as embedded-smeared crack models. In such models, all computations would be made at constitutive level and this would allow the use of standard elements with continuous displacement fields, making the implementation of these models straight-forward in any nonlinear FE code. Therefore, these would be the updated versions of the classical orthogonal crack models as introduced by Rashid in 1968 [14], but introducing the necessary corrections to avoid mesh-size and mesh-bias dependency. Following this line of thought, we present in this paper a mesh corrected crack model, formulated with the aim of covering this gap.

The objectives of this paper are threefold: (i) to formulate, at continuum level, a non-associative orthotropic Rankine damage model as the basis of the corresponding discrete crack model, (ii) to propose a mesh corrected crack model as the discrete version of the orthotropic continuum damage model, and (iii) to assess the performance 
of the proposed crack model by means of selected numerical examples which exhibit tensile cracking.

The outline of the paper is as follows. In the next Section, we briefly review the main features of crack models as used in the last decades, both at continuum and discrete levels. Then, a non-associative orthotropic Rankine continuum damage model, suitable for degradation under tensile straining, is presented. Later, the corresponding implementation of the damage model in a discrete FE framework is discussed. Finally, selected numerical examples are presented to assess the present formulation and to show the attained benefits as compared to the "straight" use of the standard orthotropic and isotropic damage based crack models.

\section{Discontinuous and continuous, true and embedded crack approaches \\ crack approaches}

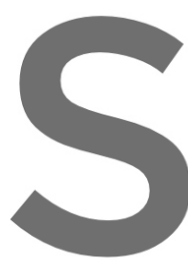

Sinee the earliest applications of the FEM to concrete structures, back in the 1960's, the modelling of cracks has been a hot topic in the FE literature, both professional and academic. Many approaches have been suggested and new terminology has been coined. In this Section we distinguish between the formulation at continuum level and

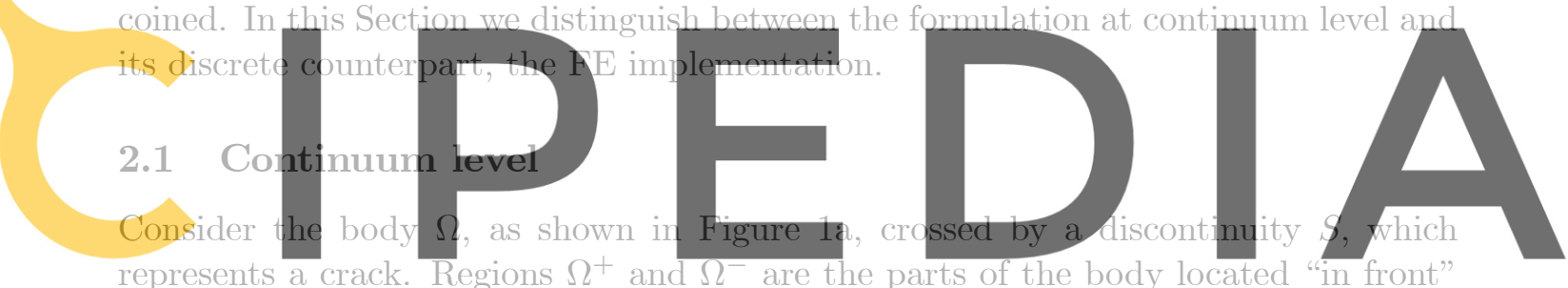

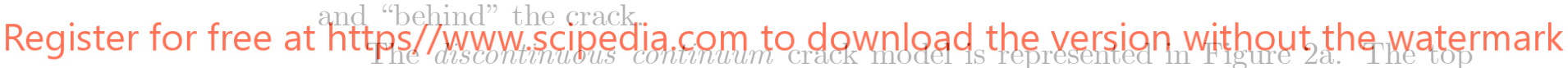

graph shows the normal displacement along a line normal to the crack, with a dis-

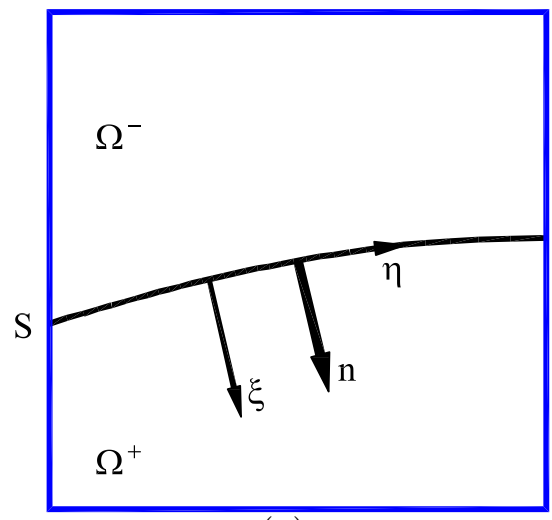

(a)

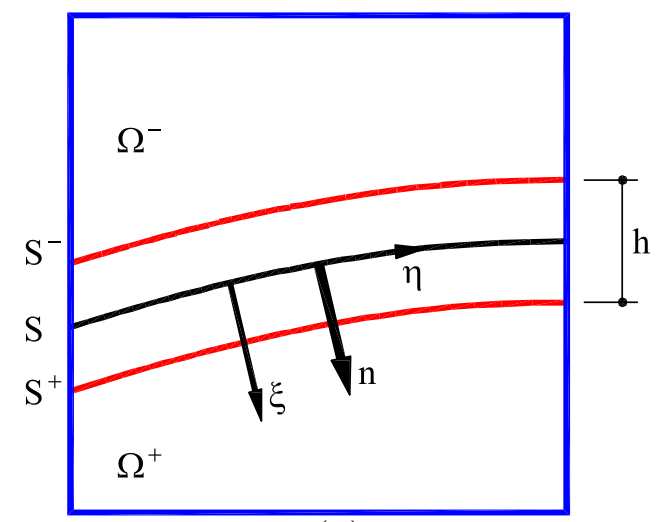

(b)

Figure 1: Modellization of a crack at continuum level: (a) discontinuous approach, (b) continuous (smeared) approach 

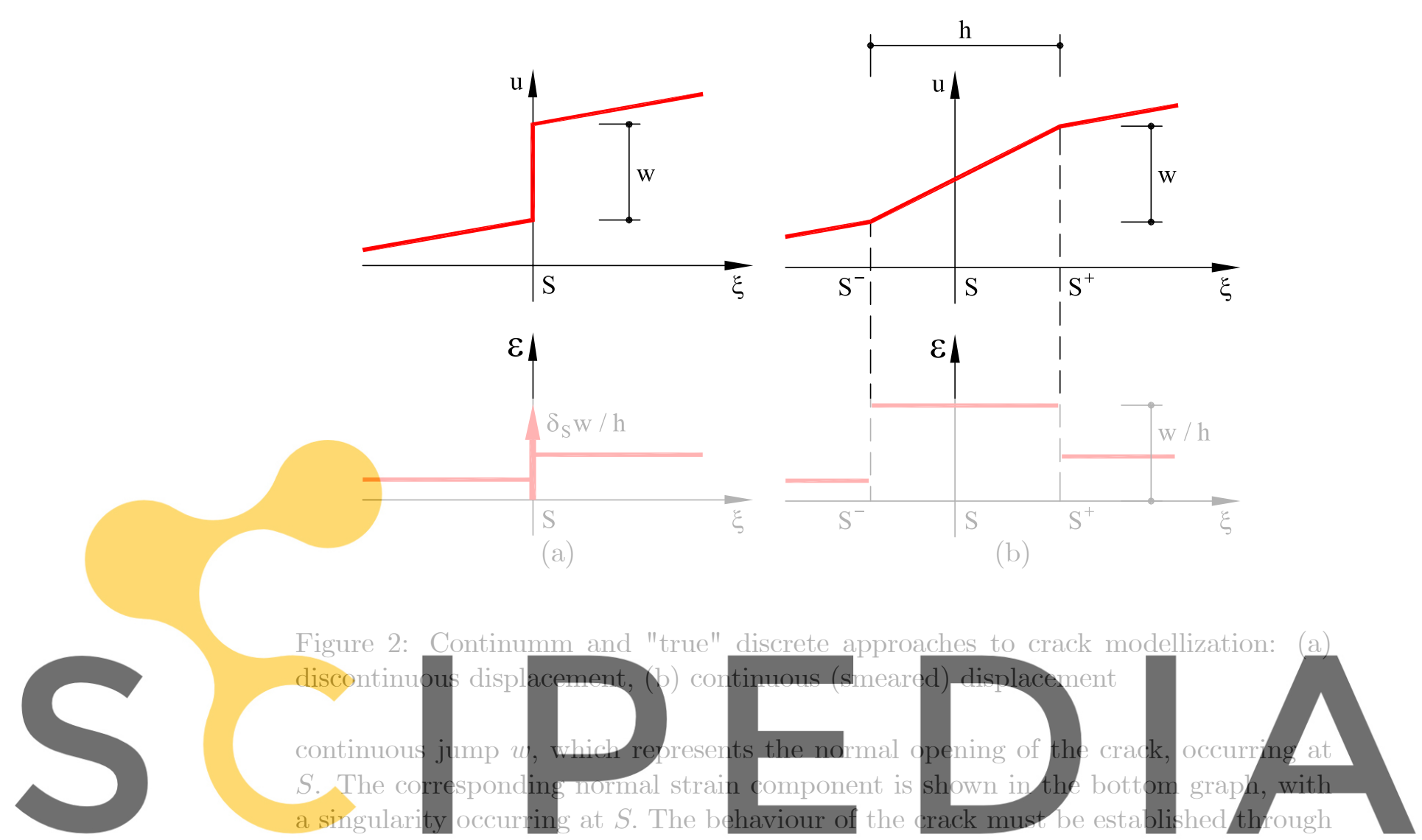

a softening traction-jump law.

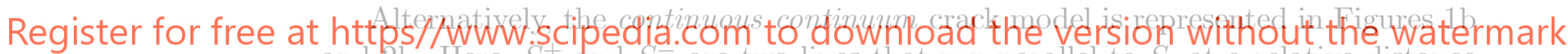

and 2 b. Here, $S^{+}$and $S^{-}$are two lines that run parallel to $S$, at a relative distance

$h$. In this model, the normal jump $w$ occurring at $S$ is smeared over the distance

$h$. The top graph in Figure 2b shows the normal displacement along a line normal to the crack, with the normal jump $w$ smeared continuously between $S^{-}$and $S^{+}$. The corresponding normal strain is shown in the bottom graph, with no discontinuity occurring at $S$. The behaviour of the crack can be established through a softening stress-(total) strain law.

\section{$2.2 \quad$ Discrete level}

Let us now consider a FE discretization of the body $\Omega$, as shown in Figure 3, crossed by a discontinuity $S$. There is the option of discretizing both the discontinuous or the continuous continuum approaches.

The true discontinuous discrete crack model reproduces the behaviour of Figure 2a. Cracks are modelled by separation of nodal points initially occupying the same spatial position, with the obvious restriction that cracks can only form along the element boundaries (Fig. 3a). This was the model adopted in the earliest applications 

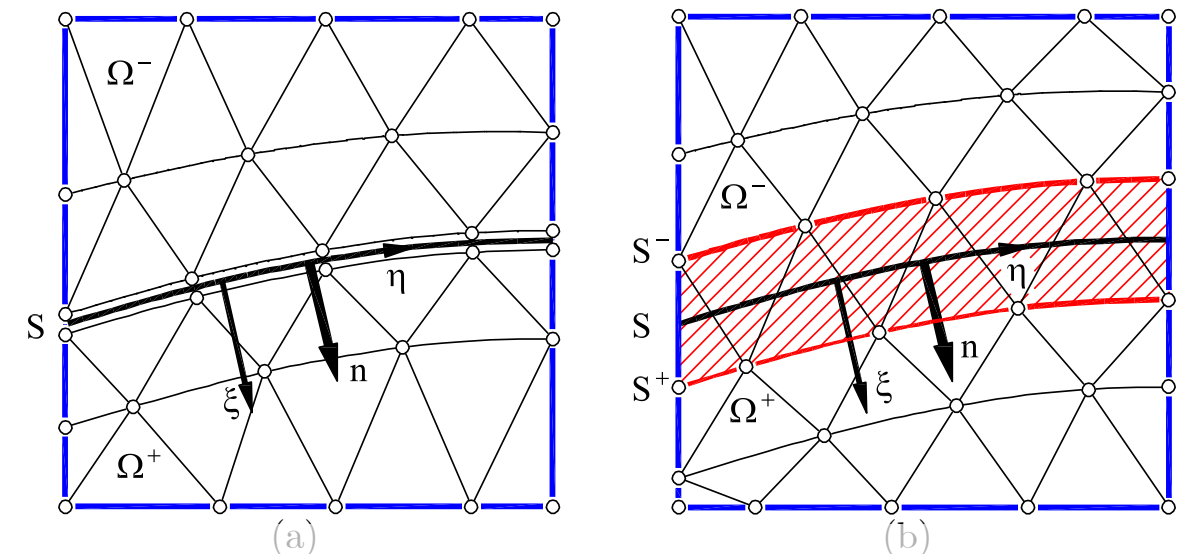

Figure 3: Modellization of a crack at discrete level: (a) discontinuous approach, (b) continuous (smeared) approach

of the FEM to concrete structures, back in the 1960's ([15], [16], [17]) and it is still widely favored in Computational Fracture Mechanics.

Alternatively, the true continuous discrete crack model reproduces the behaviour

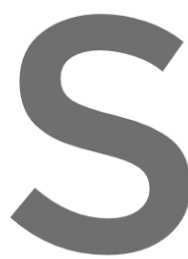

of Figure 2b. In this

to $S$, at a

elements loca

Rashid in his

As the behaviour
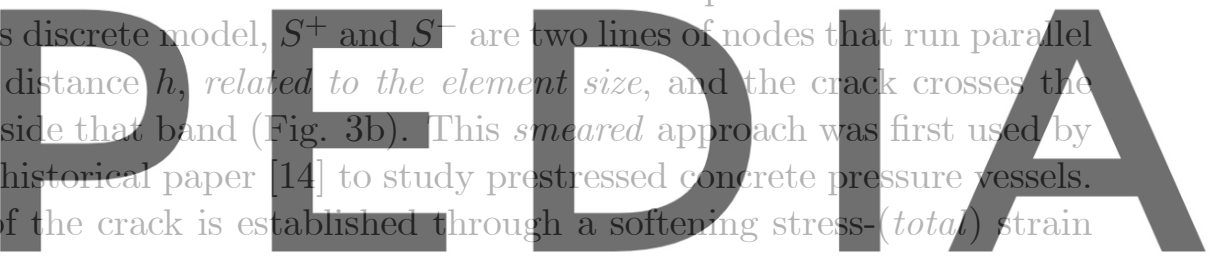

law, this approach can be implemented in any nonlinear FE code by simply writing a

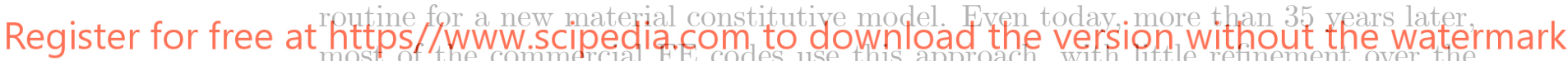

original Rashid's ideas.

However, this two options, traditional in the $\mathrm{FE}$ literature, do not exhaust all the possibilities for discrete FE crack models. Let us consider two more:

The embedded-discontinuous discrete crack model incorporates discontinuous displacement fields inside the finite elements crossed by the discontinuity in order to reproduce the displacement and strain behaviour of its continuum parent model, as shown in Figure 4a. The displacement field inside the affected elements is assumed to be discontinuous, and the strain field is decomposed into a regular part, outside the crack, and a singular part at the crack. This is the idea behind the so-called strong discontinuity approach ([4], [5], [6], [7], [8], [9], [5], [10], [11], [12], [13]). A similar idea supports the so-called extended finite element method (X-FEM) ([1], [2], [3]), which is based on enriching the nodal degrees of freedom with new ones that represent the displacement jumps across the crack.

The main disadvantage of this approach is that it requires special integration rules inside the affected finite elements to take into account what happens at and outside the discontinuity. At the discontinuity, the natural option in this embedded- 

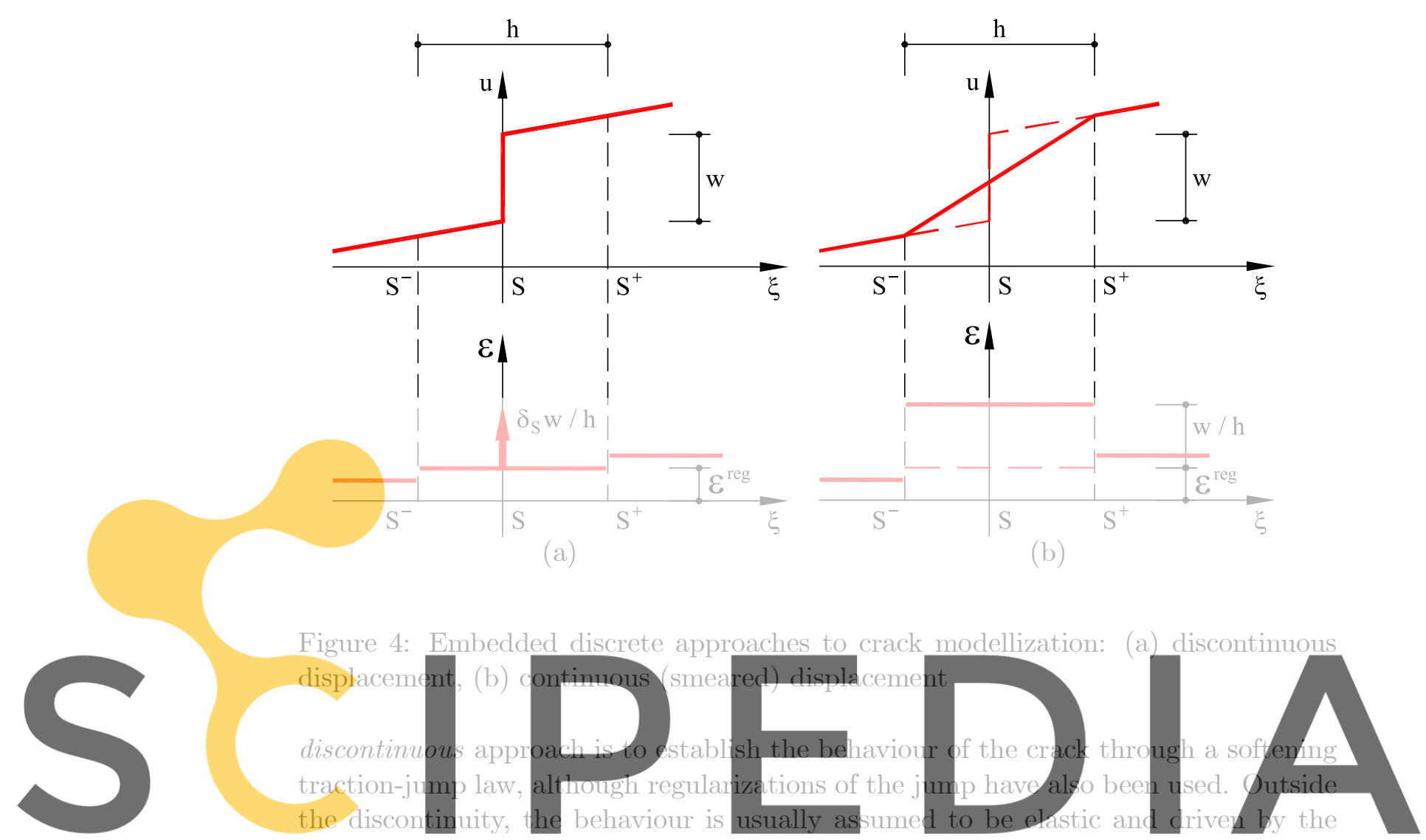

regular part of the strain in the affected elements.

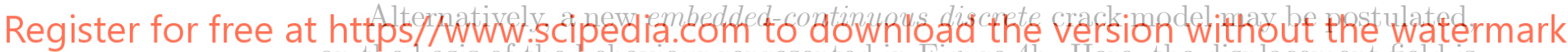

on the basis of the behaviour represented in Figure $4 \mathrm{~b}$. Here, the displacement field is

continuous in the affected elements but the strain field is decomposed into its elastic

and inelastics part, the latter due to the crack. Once the inelastic part is discounted, the behaviour is elastic. The contribution of the crack to the displacement field can be evaluated from the corresponding component of the inelastic part of the strain field.

The advantages of this novel approach are, on one hand, that the treatment of the crack is formulated at constitutive level in a stress-strain format and standard integration rules are maintained (as in the smeared approaches) and, on the other, that the decomposition of the strains into elastic and inelastic parts allows the a posteriori identification of the displacement jump (as in the discontinuous approaches).

\subsection{The discretization error}

At first sight, the previous discussion may seem rather scholastic and speculative. Certainly, the difference between the models in Figures $4 \mathrm{a}$ and $4 \mathrm{~b}$ is subtle. Even the difference between the models in Figure 4 and their simpler counterparts of Figure 2 may seem of little practical relevance. However, such point of view must be revised, 
as the pertinence of the different models can not be fully apprehended unless their application in $2 \mathrm{D}$ or $3 \mathrm{D}$ situations is considered.

In the first place, the application of the true discontinuous discrete crack model of Figure 2a requires the use of remeshing techniques, and this motivates the apparition of the embedded-discontinuous discrete crack models of Figure 4a, which still require the use of non-standard integration techniques. This is a significant drawback for the practical implementation and dissemination of these models.

Contrariwise, the true continuous discrete crack model of Figure $2 \mathrm{~b}$ is of straightforward implementation and does not require remeshing but, unfortunately, in a multidimensional setting, its original format based on orthotropic models is known to suffer from serious stress locking. In reference [18], the detailed analysis of the discrete rotating crack model reveals that it necessarily leads to the development of spurious shear strains which grow linearly with the crack opening, inducing stress locking. This evidence led in the 90's to the progressive neglect of the orthotropic models in favor of isotropic models, both in damage or plasticity frameworks. Even if smeared isotropic models largely mitigate the problem of stress locking, they cannot reproduce locally the directional nature of cracking.

In any case, the analysis of the classical smeared models reveals the key point of

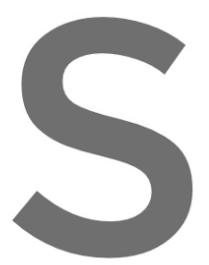

he matter: the problem does not

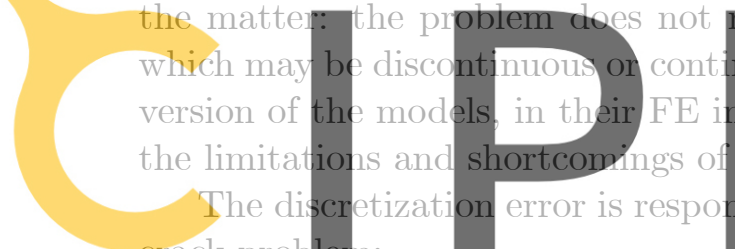

crack problem:
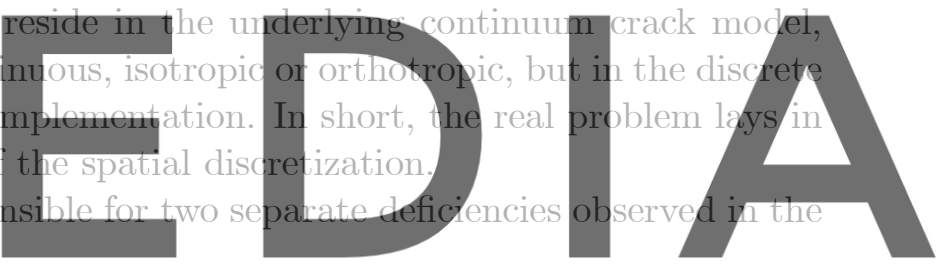

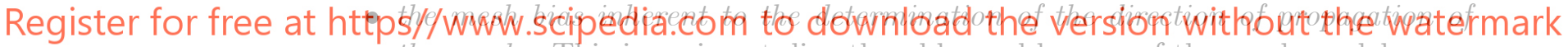

the cracks. This issue is not directly addressed by any of the crack models even if all of them suffer from it. It is not yet generally realized that this is a problem of the discrete model and not of the continuum model. Tracking algorithms, formulated outside the constitutive model, are a partial solution, but they are not robust enough in certain situations, like bending [19], [20].

- the inappropriate modelling of the inelastic strain and stress fields associated to cracking. This issue is directly related to the stress locking observed in the classical orthotropic crack models and it applies even if the crack path is known in advance. In this regard, much knowledge has been accumulated in the last decade through the use of the embedded-discontinuous discrete crack models. In this work, we intend to transfer this know-how to the formulation of embeddedcontinuous discrete crack models.

The situation described is similar to what happens when using incompressible von Mises type softening models, either in a plasticity or damage format. In those cases, the discrete problem has to be modified appropriately, or "mesh corrected", to obtain mesh independent results [21], [22]. 


\section{Orthotropic Rankine Damage Model}

\subsection{Fixed and rotating orthotropic Rankine models}

Continuum damage models have been frequently used in the last 20 years to simulate tensile fracture, although, commonly, isotropic models were used ([19], [20], [23], [24], [25], [26]). However, anisotropic models have also been formulated [27]. In fact, the framework of Continuum Damage Mechanics is very similar to the format of the original smeared crack models [28].

In this Section we define a non-associative orthotropic damage model which depends on the definition of two physical directions aligned with two unit vectors $\mathbf{n}$ and $m$. Vector $n$ is orthogonal to the damaged plane and vector $m$ determines the structure of the inelastic strains.

For a classical Rankine model, damage occurs in a plane orthogonal to the major principal strain/stress and, therefore, this is the direction of vector $\mathbf{n}$. It is also standard, at continuum level, to consider associative behaviour, so $\mathbf{m}=\mathbf{n}$. As it will be shown below, the need for a non-associative model, with $\mathbf{m} \neq \mathbf{n}$, arises in the discrete problem, when the behaviour of finite elements is considered.

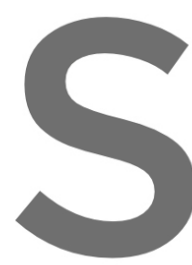

As a Ran it has beet linine

ception of damage a co-rotational assc plane. It has been was soon dbserved
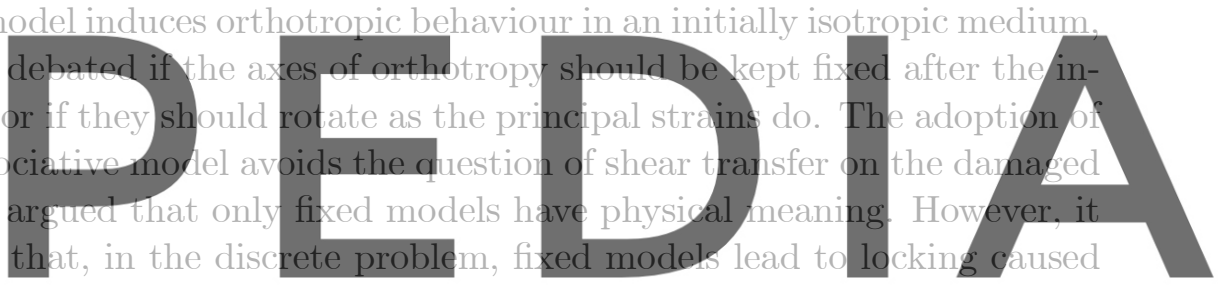

by spurious shear strains. This has favored the use of rotating models and, even,

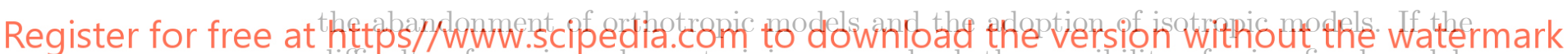
difficulty of spuriouts shear straining was solved, the possibility of using fixed models could be considered again.

\subsection{Inelastic strains and deformation}

Let $\boldsymbol{\varepsilon}$ be the total strain tensor, computed as $\varepsilon=\nabla^{s} \mathbf{u}$, where $\mathbf{u}$ are the displacements, and let $\mathbf{m}$ be a unit vector associated to a physical direction in space. The deformation vector in this direction is defined as:

$$
\delta=\varepsilon \cdot \mathbf{m}=\mathbf{M}: \varepsilon
$$

where $\mathbf{M}$ is a third order tensor defined as $M_{i j k}=\frac{1}{2}\left(\delta_{j k} m_{i}+\delta_{i k} m_{j}\right)$ from the components of vector $\mathbf{m}$. The symbols $(\cdot)$ and (:) denote simple and double contractions, respectively.

Consider the usual decomposition of the total strain tensor into its elastic and inelastic components:

$$
\varepsilon=\varepsilon^{e}+\varepsilon^{i}
$$


As our aim is to define an orthotropic damage model, let us define the following structure for the inelastic strain tensor:

$$
\varepsilon^{i}=(\mathbf{m} \otimes \mathbf{e})^{S}=\mathbf{M} \cdot \mathbf{e}
$$

where $\mathbf{m}$ is a unit vector pointing in a physical direction to be defined and $\mathbf{e}$ is an inelastic deformation vector. The symbol $(\otimes)$ denotes the tensor product and $(\cdot)^{S}=\operatorname{symm}(\cdot)$. Tensor $\mathbf{M}$ in Eq. (3) has the same structure as the one in Eq. (1).

\subsection{Constitutive Equation}

In a strain-based formulation, a continuum damage model is usually based on the definition of the effective stress, which is introduced in connection with the hypothesis of strain equivalence [29]: the strain associated with a damaged state under the applied stress $\sigma$ is equivalent to the strain associated with its undamaged state under the effective stress $\bar{\sigma}$. In the present work, the effective stresses $\overline{\boldsymbol{\sigma}}$ is computed in terms of the total strain tensor $\varepsilon$ as

$$
\bar{\sigma}=\mathbf{C}: \varepsilon
$$

where $\mathbf{C}$ is the usual (fourth order) isotropic linear-elastic constitutive tensor.

The constitutive equation for the orthotropic damage model is defined as:

$$
\boldsymbol{\sigma}=\left(\mathbf{I}_{4}-\mathbf{D}\right): \overline{\boldsymbol{\sigma}}=\left(\mathbf{I}_{4}-\mathbf{D}\right): \mathbf{C}: \boldsymbol{\varepsilon}
$$

where $\mathbf{I}_{4}$ is a symmetric fourth order unit tensor $\left(\mathbf{I}_{4}: \mathbf{C}=\mathbf{C}, I_{i j k l}=\frac{1}{2}\left(\delta_{i k} \delta_{j l}+\delta_{i l} \delta_{j k}\right)\right)$ and we have introduced $\mathbf{D}$, the fourth order damage tensor, whose definition and evolution is given below.

Introducing the strain decomposition in Eq. (2), we can write:

$$
\boldsymbol{\sigma}=\overline{\boldsymbol{\sigma}}-\boldsymbol{\sigma}^{i}=\mathbf{C}:\left(\varepsilon-\varepsilon^{i}\right)
$$

\subsection{Non-Associative Orthotropic Damage}

The evolution of the orthotropic damage tensor $\mathbf{D}$ will be associated to a physical direction in space identified by a unit vector $\mathbf{n}$ and the plane orthogonal to it. The traction vector acting on this plane is:

$$
\mathbf{t}=\boldsymbol{\sigma} \cdot \mathbf{n}=\mathbf{N}: \boldsymbol{\sigma}
$$

where $\mathbf{N}$ is a third order tensor defined as $N_{i j k}=\frac{1}{2}\left(\delta_{j k} n_{i}+\delta_{i k} n_{j}\right)$ from the components of vector $\mathbf{n}$. Note than tensor $\mathbf{N}$ has the same structure as $\mathbf{M}$ in Eq. (1).

Using Eqs. (7), (6) and (3), we can write:

$$
\begin{aligned}
\mathbf{t} & =\mathbf{N}: \boldsymbol{\sigma} \\
& =\mathbf{N}:\left[\mathbf{C}: \boldsymbol{\varepsilon}-\mathbf{C}: \varepsilon^{i}\right] \\
& =\mathbf{N}:[\mathbf{C}: \boldsymbol{\varepsilon}-\mathbf{C}: \mathbf{M} \cdot \mathbf{e}] \\
& =\overline{\mathbf{t}}-[\mathbf{N}: \mathbf{C}: \mathbf{M}] \cdot \mathbf{e}
\end{aligned}
$$


where we have introduced the effective traction vector $\overline{\mathbf{t}}=\overline{\boldsymbol{\sigma}} \cdot \mathbf{n}=\mathbf{N}: \overline{\boldsymbol{\sigma}}$. Note that $\mathbf{N}: \mathbf{C}: \mathbf{M}=\mathbf{n} \cdot \mathbf{C} \cdot \mathbf{m}$.

To proceed, the traction vs inelastic deformation, $\mathbf{t}$ vs $\mathbf{e}$, relationship must be established. This is the continuum equivalent of a discrete traction-separation law. We can write, for instance,

$$
\mathbf{t}=\widehat{\boldsymbol{\Gamma}}^{-1} \cdot \widehat{\mathbf{C}} \cdot \mathbf{e}=\widehat{\mathbf{H}} \cdot \mathbf{e}
$$

where $\widehat{\boldsymbol{\Gamma}}$ is a second order symmetric traction compliance tensor, which may be either a function of the traction or the inelastic deformation vector, and $\widehat{\mathbf{C}}$ is a second order symmetric reference stiffness tensor. Substituting law (9) into the equilibrium condition (8d) leads to:

$$
\mathbf{e}=[\widehat{\mathbf{H}}+\mathbf{N}: \mathbf{C}: \mathbf{M}]^{-1} \cdot \overline{\mathbf{t}}=\widehat{\mathbf{A}} \cdot \overline{\mathbf{t}}
$$

Symmetry arguments lead to the condition that, in local coordinates for which the first axis is aligned with vector $\mathbf{n}$, the reference stiffness tensor must be diagonal. Therefore, a natural option is to take $\widehat{\mathbf{C}}=\mathbf{N}: \mathbf{C}: \mathbf{N}$, which relates the reference stiffness of the crack to the elastic moduli of the undamaged material.

Note that as the traction compliance tensor is defined as a function of the traction, $\widehat{\boldsymbol{\Gamma}}=\widehat{\boldsymbol{\Gamma}}(\mathbf{t})$, or of the inelastic deformation vector, $\widehat{\boldsymbol{\Gamma}}=\widehat{\boldsymbol{\Gamma}}(\mathbf{e})$, Eq. (10) is non linear, and it has to be solved iteratively.

Once the inelastic deformation vector is known, Eq. (3) defines the inelastic strain tensor as

$$
\varepsilon^{i}=\mathbf{M} \cdot \mathbf{e}=\mathbf{M} \cdot \widehat{\mathbf{A}} \cdot \overline{\mathbf{t}}=(\mathbf{M} \cdot \widehat{\mathbf{A}} \cdot \mathbf{N}): \overline{\boldsymbol{\sigma}}=(\mathbf{M} \cdot \widehat{\mathbf{A}} \cdot \mathbf{N}): \mathbf{C}: \varepsilon
$$

and the inelastic stress tensor is then

$$
\boldsymbol{\sigma}^{i}=\mathbf{C}: \varepsilon^{i}=\mathbf{C}:(\mathbf{M} \cdot \widehat{\mathbf{A}} \cdot \mathbf{N}): \mathbf{C}: \boldsymbol{\varepsilon}
$$

Relating Eq. (12) to its definition in (5), the damage tensor is finally obtained as

$$
\mathbf{D}=\mathbf{C}:(\mathbf{M} \cdot \widehat{\mathbf{A}} \cdot \mathbf{N})
$$

Note that the damage tensor $\mathbf{D}$ is symmetric only if the direction $\mathbf{m}$ that defines the structure of the inelastic strains coincides with the direction $\mathbf{n}$ that defines the tractions, so that $\mathbf{M}=\mathbf{N}$. For that particular case, we can define the model as associative. Otherwise, the model is non-associative.

\subsection{The traction compliance and damage tensors}

It is clear from the previous Section that the essence of the orthotropic damage model lays in the definition of the traction compliance tensor, as this defines the relation between the inelastic deformation and the traction acting on the plane orthogonal 
to vector $\mathbf{n}$. In the context of a damage model, it is natural to define this relation in terms of damage rather than compliance. The traction compliance tensor can be related to a corresponding traction damage tensor defined as

$$
\widehat{\mathbf{D}}=\left[\mathbf{I}+\widehat{\boldsymbol{\Gamma}}^{-1}\right]^{-1}
$$

so that

$$
\widehat{\boldsymbol{\Gamma}}^{-1}=\widehat{\mathbf{D}}^{-1}-\mathbf{I}
$$

For the definition of these tensors, it is convenient to work in an orthonormal basis in which the first direction coincides with $\mathbf{n}$. We will refer to this basis as natural basis in the following.

In the natural basis, the simplest traction damage and compliance tensors take the diagonal forms

$$
\widehat{\mathbf{D}}=d \mathbf{I} \quad, \quad \widehat{\boldsymbol{\Gamma}}=\frac{d}{1-d} \mathbf{I}
$$

where $d$, the damage index, is a scalar internal-like variable whose definition and evolution is discussed below.

This simple definition of $\widehat{\mathbf{D}}$ implies that the normal and tangential components of the traction degrade in the same way. The model may be refined by adopting a still diagonal form for $\widehat{\mathbf{D}}$, but with different damage indices, $d_{n}$ and $d_{t}$, for the normal and tangential components, respectively. The tangential damage index can be a explicit function of the normal damage index, $d_{t}=d_{t}\left(d_{n}\right)$.

Using Eq. (16) and $\widehat{\mathbf{C}}=\mathbf{N}: \mathbf{C}: \mathbf{N}$, tensor $\widehat{\mathbf{A}}=\left[\frac{1}{d} \widehat{\mathbf{C}}+\mathbf{N}: \mathbf{C}:(\mathbf{M}-\mathbf{N})\right]^{-1}$ and the damage tensor is

$$
\mathbf{D}=\mathbf{C}:\left(\mathbf{M} \cdot\left[\frac{1}{d} \widehat{\mathbf{C}}+\mathbf{N}: \mathbf{C}:(\mathbf{M}-\mathbf{N})\right]^{-1} \cdot \mathbf{N}\right)
$$

Note that if $\mathbf{M}=\mathbf{N}$,

$$
\mathbf{D}=d\left[\mathbf{C}:\left(\mathbf{N} \cdot \widehat{\mathbf{C}}^{-1} \cdot \mathbf{N}\right)\right]
$$

This can be recognized as a standard definition of an orthogonal damage model.

\subsection{Isotropic damage model}

It is convenient to recall that the orthotropic nature of the proposed model resides in the structure of the inelastic component of the strains, Eq. (3). The structure of the model changes by changing this. For instance, the standard isotropic damage model is recovered by postulating that the inelastic strain tensor is proportional to the total strain:

$$
\varepsilon^{i}=d \varepsilon
$$

which corresponds to a diagonal damage tensor $\mathbf{D}=d \mathbf{I}_{4}$. 


\subsection{The damage index}

In order to define a damage model sensitive only to tensile stresses, we define the equivalent stress in the form:

$$
\tau=\left\langle\sigma_{n}\right\rangle=\langle\mathbf{n} \cdot \boldsymbol{\sigma} \cdot \mathbf{n}\rangle
$$

where $\sigma_{n}$ is the normal stress acting on the plane orthogonal to direction $\mathbf{n}$. The symbols $\langle\cdot\rangle$ are the Macaulay brackets $(\langle x\rangle=x$, if $x \geq 0,\langle x\rangle=0$, if $x<0)$.

With this definition for the equivalent stress, the damage criterion, $\Phi$, is introduced as:

$$
\Phi(\tau, q)=\tau-q(r) \leq 0
$$

where the function $q=q(r)$ is the stress-like softening function. It is clear that the definition in Eq. (21) corresponds to the Rankine criterion. Figure 5a shows a schematic representation of the defined damage criterion in the stress space.

Variable $r$ is an internal stress-like variable representing the current damage threshold, as its value controls the size of the damage surface. The initial value of the damage threshold is $r_{o}=\sigma_{o}$, where $\sigma_{o}$ is the initial uniaxial damage stress.

In this work, we will use following softening laws $q=q(r)$ :

- Linear softening:

$$
q(r)=\left\{\begin{array}{cl}
r_{o}-H_{S}\left(r-r_{o}\right) & r_{o} \leq r \leq r_{u} \\
0 & r \geq r_{u}
\end{array}\right.
$$

- Exponential softening:

$$
q(r)=r_{o} \exp \left\{-2 H_{S}\left(\frac{r-r_{o}}{r_{o}}\right)\right\} \quad r_{o} \leq r
$$

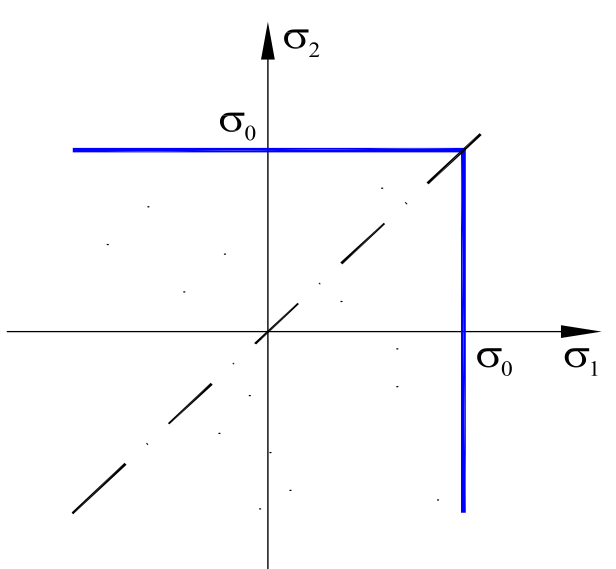

(a)

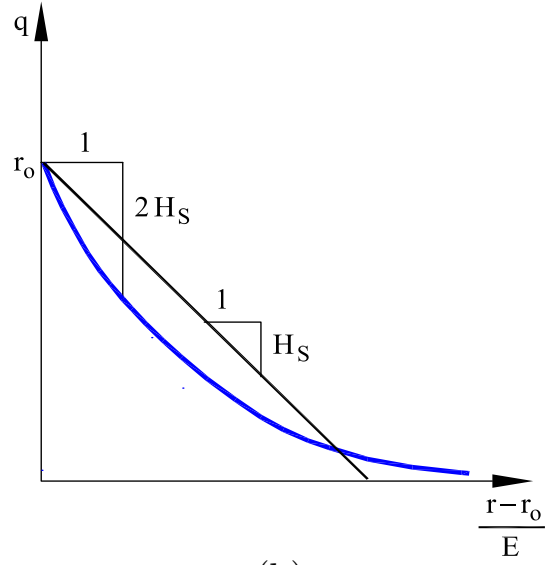

(b)

Figure 5: Rankine damage model: (a) damage surface, (b) softening functions 
where $H_{S} \geq 0$ is a constant. Figure $5 \mathrm{~b}$ shows a schematic representation of both these functions.

The damage index $d=d(r)$ is explicitly defined in terms of the corresponding current value of the damage threshold

$$
d(r)=1-\frac{q(r)}{r} \quad r_{o} \leq r
$$

so that it is a monotonically increasing function such that $0 \leq d \leq 1$.

The evolution of the damage bounding surface for loading, unloading and reloading conditions is controlled by the Kuhn-Tucker relations and the damage consistency condition, which are

$$
\begin{aligned}
& \dot{r} \geq 0 \quad \Phi(\tau, r) \leq 0 \quad \dot{r} \Phi(\tau, r)=0 \\
& \text { if } \Phi(\tau, r)=0 \text { then } \dot{r} \dot{\Phi}(\tau, r)=0
\end{aligned}
$$

Because the value of the normal stress in (21) depends on the damage tensor and this, in turn, depends on the damage index, these equations must be solved iteratively at the same time as the equilibrium equation (10).

\subsection{Mechanical dissipation}

For the associative orthotropic damage model, the mechanical free energy is defined in the form:

$$
W=\frac{1}{2} \varepsilon:\left(\mathbf{I}_{4}-\mathbf{D}\right): \mathbf{C}: \boldsymbol{\varepsilon} \geq 0
$$

where the damage tensor $\mathbf{D}$ is symmetric. The condition $W \geq 0$ must be verified for any given structure of tensor $\mathbf{D}$. The condition is verified if $\mathbf{D}$ is positive definite and $\|\mathbf{D}\| \leq 1$. From Eq. (13), taking $\mathbf{M}=\mathbf{N}$, it is

$$
\mathbf{D}=\mathbf{C}:(\mathbf{N} \cdot \widehat{\mathbf{A}} \cdot \mathbf{N})
$$

so, the necessary conditions will verify if $\widehat{\mathbf{A}}$ is positive definite and $\|\mathbf{A}\| \leq 1$. From Eqs. (9) and (10), and taking $\widehat{\mathbf{C}}=\mathbf{N}: \mathbf{C}: \mathbf{N}$, it is

$$
\widehat{\mathbf{A}}=\left[\widehat{\mathbf{D}}^{-1} \cdot \widehat{\mathbf{C}}\right]^{-1}=\widehat{\mathbf{C}}^{-1} \cdot \widehat{\mathbf{D}}
$$

so, $\widehat{\mathbf{D}}$ must be positive definite and $\|\widehat{\mathbf{D}}\| \leq 1$.

From expression (26), the constitutive equation is obtained, applying Coleman's method, as

$$
\boldsymbol{\sigma}=\left(\mathbf{I}_{4}-\mathbf{D}\right): \mathbf{C}: \boldsymbol{\varepsilon}
$$

Also, by the same procedure, the rate of mechanical dissipation is obtained as

$$
\dot{\mathcal{D}}=\frac{1}{2} \varepsilon: \dot{\mathbf{D}}: \mathbf{C}: \boldsymbol{\varepsilon} \quad \geq 0
$$


The condition $\dot{\mathcal{D}} \geq 0$ will be verified provided that the rate of the damage tensor $\dot{\mathbf{D}}$ is positive definite. In view of Eqs. (27) and (28), this reduces to the condition that the rate of the traction damage tensor $\dot{\hat{\mathbf{D}}}$ must be positive definite.

The necessary conditions on $\widehat{\mathbf{D}}$ and $\dot{\hat{\mathbf{D}}}$ depend on the actual definition of $\widehat{\mathbf{D}}$. For the simple case $\widehat{\mathbf{D}}=d \mathbf{I}$, it is $\dot{\hat{\mathbf{D}}}=\dot{d} \mathbf{I}$ and, therefore, the conditions reduce to $0 \leq d \leq 1$ and $\dot{d} \geq 0$.

For the non-associative orthotropic damage model, $\mathbf{D}$ is not symmetric, so the constitutive relationship (29) cannot be derived from a potential like in the associative case. However, by analogy, we will define the rate of mechanical dissipation in the same form as in Eq. (30). Now, the extra condition $\mathbf{m} \cdot \mathbf{n} \geq 0$, together with the already stated conditions that $\widehat{\mathbf{D}}$ must be positive definite and $\|\widehat{\mathbf{D}}\| \leq 1$, it is sufficient to ensure that $\dot{\mathcal{D}} \geq 0$.

\subsection{Strain-softening and fracture energy release}

In order to relate the specific dissipated energy $\mathcal{D}$, defined per unit volume, to the mode I fracture energy of the material $\mathcal{G}_{f}$, defined per unit area of damaged material, let us introduce a characteristic length $h$, such that

$$
\mathcal{D} h=\mathcal{G}_{f}
$$

This makes the softening modulus $H_{S}$, which defines the softening response, dependent on this length.

Let us now consider an uniaxial tensile experiment in which the tensile strain increases monotonically and quasi-statically from an initial unstressed state to another in which full degradation takes place. The specific energy dissipated in the process is:

$$
\mathcal{D}=\int_{t=0}^{t=\infty} \dot{\mathcal{D}} d t=\frac{1}{E} \int_{r=r_{o}}^{r=\infty} q d r
$$

where $E$ is the Young's modulus. Using Eqs. (22) and (23), it can be shown that both for the linear and exponential softening cases, it results

$$
\mathcal{D}=\left(1+\frac{1}{H_{S}}\right) \frac{\sigma_{o}^{2}}{2 E}
$$

and equating $\mathcal{D}=\mathcal{G}_{f} / h$, we have

$$
H_{S}=\frac{\bar{H}_{S} h}{1-\bar{H}_{S} h} \quad \geq 0
$$

where $\bar{H}_{S}=\sigma_{o}^{2} /\left(2 E \mathcal{G}_{f}\right)$ depends only on the material properties. Defining the material length $\frac{o}{l_{S}}=1 / \bar{H}_{S}$, Eq. (34) can be rewritten as

$$
H_{S}=\frac{h}{\overline{l_{S}}-h} \simeq \frac{h}{\overline{l_{S}}}
$$


where the approximation holds for $\overline{l_{S}} \gg h$.

It must be remarked that the above computation of the total specific dissipation has been obtained for an uniaxial stress state. In a more general case, the total dissipated energy may be larger than that in expression (33). In the associative case and a rotating model, the principal directions of strains and stress coincide and there is no additional dissipation due to shear on the damaged plane, even if these directions vary during the loading process. For the cases of fixed or non-associative models, there would be additional dissipation due to shear.

\section{The Mesh Corrected Crack Model. Finite element formulation}

In this Section we describe the implementation of the continuum orthogonal Rankine damage model described above in a FE framework. We will refer to the resulting discrete model as the mesh corrected crack model. Let us recall that in the orthotropic Rankine damage model vector $\mathbf{n}$ defines the direction for which the tractions $(\overline{\mathbf{t}}$ and t) and the deformation vector $\mathbf{e}$ are defined, while vector $\mathbf{m}$ defines the structure of the inelastic strains $\varepsilon^{i}$.

For simplicity, we will restrict our discussion to the 3-noded linear triangle (CST). Let us consider the CST shown in Figure 6. Let us assume that the triangle is crossed by a crack (discontinuity) with a unit vector $\mathbf{n}_{h}$ pointing in the direction normal to the crack. Let $\mathbf{m}_{h}$ be another unit vector which is selected as the normal to the three element sides that maximizes the value of the product $\left|\mathbf{n}_{h} \cdot \mathbf{m}_{h}\right|$. Let us call $i^{-}$and $j^{-}$to the nodes that define this side, and $k^{+}$to the remaining node. We will assume that the crack does not intersect the side $i^{-}-j^{-}$and that it separates this side from node $k^{+}$(solitary node). We direct vector $\mathbf{n}_{h}$ so that it points to $k^{+}$and vector $\mathbf{m}_{h}$ so that $\mathbf{n}_{h} \cdot \mathbf{m}_{h}>0$.

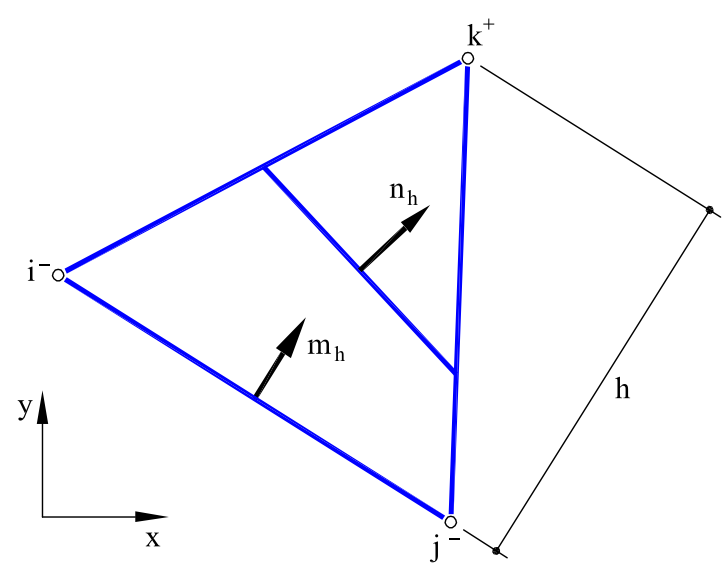

Figure 6: Implementation of the Mesh Corrected Crack Model in a CST triangle 
In the FE implementation of the embedded-continuous damage model we will take the width of the localization band, $h$, as the distance from the solitary node $k^{+}$to the opposite side $i^{-}-j^{-}$. This allows to identify the contribution of the crack to the displacement field inside the element as $\mathbf{w}=h \mathbf{e}$. Note that this "displacement jump" is not co-axial with vector $\mathbf{n}$, nor with vector $\mathbf{m}$. The appropriate selection of length $h$ is very important in the FE implementation of a smeared model, as it determines the discrete softening parameter, $H_{S}$ in Eq. (35). In an orthotropic model, where damage is associated to a specific direction of stress/strain, $h$ must be selected according to this. In an isotropic model, it is usual to select length $h$ related to the element diameter. However, in this work, the same determination of $h$ as for the orthotropic model will be used for comparison.

With these definitions we can define three different crack models as the discrete versions of different orthotropic Rankine damage models:

- Take $\mathbf{n}=\mathbf{n}_{h}$ and $\mathbf{m}=\mathbf{n}_{h}$. This model is statically consistent and symmetric and it is often referred to as SOS (statically optimal symmetric) in the literature of embedded-discontinuous models [7]. It is totally equivalent to the classical fixed or rotating crack models and, therefore, it can be shown to lock due to spurious shear (see [18]).

- Take $\mathbf{n}=\mathbf{m}_{h}$ and $\mathbf{m}=\mathbf{m}_{h}$. This model is kinematically consistent with the spatial discretization and symmetric and it is often referred to as KOS (kinematically optimal symmetric) in the literature of embedded-discontinuous models. It does not lock, but it does not satisfy the consistency condition for the stresses on the surface of the discontinuity.

- Take $\mathbf{n}=\mathbf{n}_{h}$ and $\mathbf{m}=\mathbf{m}_{h}$. This model is statically and kinematically consistent but it is non-symmetric and it is often referred to as SKON (statically and kinematically optimal non-symmetric) in the literature of embedded-discontinuous models. It does not lock and it satisfies the consistency condition for the stresses on the surface of the discontinuity. We will refer to this model as Mesh Corrected Crack Model.

Using the standard matrix notation, we can write the internal force vector for the element $\Omega_{e}$ as:

$$
\mathbf{f}^{\text {int }}=\int_{\Omega_{e}} \mathbf{B}^{T} \boldsymbol{\sigma} d \Omega_{e}=\mathbf{B}^{T} \boldsymbol{\sigma} A_{e}
$$

where $\mathbf{B}$ is the usual displacements-strain matrix, $\boldsymbol{\sigma}$ is the stress vector and $A_{e}$ is the element area. For the CST, B and $\boldsymbol{\sigma}$ are constant over the element. Using the constitutive relationship

$$
\boldsymbol{\sigma}=\mathbf{C}^{\mathrm{sec}} \boldsymbol{\varepsilon}=\mathbf{C}^{\mathrm{sec}} \mathbf{B} \mathbf{d}
$$

where $\mathbf{C}^{\text {sec }}=(\mathbf{I}-\mathbf{D}) \mathbf{C}, \boldsymbol{\varepsilon}$ is the strain vector and $\mathbf{d}$ is the vector of nodal displacements. $\mathbf{C}$ is the elastic constitutive matrix and $\mathbf{D}$ is the damage matrix. Therefore:

$$
\mathbf{f}^{\mathrm{int}}=\left[\mathbf{B}^{T} \mathbf{C}^{\mathrm{sec}} \mathbf{B} A_{e}\right] \mathbf{d}
$$


This can also be written as

$$
\mathbf{f}^{\text {int }}=\left[\mathbf{B}^{T} \mathbf{C} \overline{\mathbf{B}} A_{e}\right] \mathbf{d} \quad \text { with } \quad \overline{\mathbf{B}}=\left(\mathbf{I}-\mathbf{C}^{-1} \mathbf{D C}\right) \mathbf{B}
$$

which sets the present discrete model inside the framework of the enhanced assumed strain (EAS) or B-bar methods.

\section{Numerical examples}

The formulation presented in the preceding sections is illustrated below by solving two different benchmark problems. Performance of the standard continuous displacement finite elements is tested considering $2 D$ plane-stress 3-noded linear triangular meshes. As the study of the problem of crack propagation is out of the scope of this work, we will consider that the path of cracking is known in advance; due to the symmetry of the test cases considered, this is not difficult.

The discrete problem is solved incrementally, in a (pseudo)time step-by-step manner. In all cases 100 equal time steps are performed to complete the analyses. Within each step, a modified Newton-Raphson method, together with a line search procedure, is used to solve the corresponding non-linear system of equations. Convergence of a time step is attained when the ratio between the norm of the iterative and the incremental norm of the computed displacements is lower than $10^{-5}$.

Calculations are performed with an enhanced version of the finite element program COMET [30], developed by the authors at the International Center for Numerical Methods in Engineering (CIMNE). Pre and post-processing is done with GiD, also developed at CIMNE [31].

\subsection{Rectangular strip under tension}

The first example is a plane-stress rectangular strip subjected to axial vertical straining imposed at both ends. Dimensions of the strip are $100 \times 200 \mathrm{~mm} \times \mathrm{mm}$ (width $\times$ height) and the thickness of the strip is $10 \mathrm{~mm}$. The following material properties are assumed: Young's modulus $E=2 \mathrm{GPa}$, Poisson's ratio $\nu=0.3$, tensile strength $\sigma_{o}=$ $1 \mathrm{MPa}$ and mode I fracture energy $G_{f}=250 \mathrm{~J} / \mathrm{m}^{2}$.

The computational domain is discretized in two different structured meshes with different preferential alignments. Mesh A (Fig. 7a.1) consists of rectangular triangles with predominant directions at $-45^{\circ}, 0^{\circ}$ and $+90^{\circ}$ with the horizontal axis. As the strip cracks along an horizontal line, the elements in this mesh have one of their sides parallel to the opening crack. On the other hand, mesh B (Fig. 7b.1) consists mostly of equilateral triangles with predominant directions at $-30^{\circ},+30^{\circ}$ and $+90^{\circ}$ with the horizontal axis. Therefore, the elements in this mesh do not have any of their sides parallel to the opening crack. Both meshes consists of about 900 nodes and 1,800 elements, although the results obtained are independent from mesh refinement.

This example is selected because it represents a sort of patch test for pure mode I fracture, as the stress field is uniform before cracking and it should remain so after 
cracking. On the other hand, the uniform strain field before cracking bifurcates into two uniform but different strain fields inside and outside the localization band after cracking. The example is used to assess the ability of the isotropic (ISO), associative orthotropic (ORT) and mesh corrected orthotropic (MCO) damage models to reproduce these ideal conditions. Because the direction of applied straining does not change during the loading process, we will use the fixed versions of the orthotropic models, with the directions of orthotropy determined and fixed at the onset of cracking.

The expected and computed deformed shapes of the strip using meshes A and B are shown in Figures 7a.2 and 7b.2, respectively ( (half)-imposed vertical displacement $\delta=0.05 \mathrm{~mm}$, with a displacement amplification factor of 50; the other half-imposed displacement is applied at the opposite end of the strip). As shown, the opening crack in both analyses follows exactly the horizontal axis of symmetry of the strip, and the deformation mode obtained is globally correct for the three models used and both meshes. However, subtle and not so subtle differences are revealed when a detailed analysis of the results is undertaken.

Figure 8 shows the results obtained with the three constitutive models using the well-aligned mesh A. The top plot shows the load vs displacement curve, identical for the three models and coincident with the analytical response. The middle plot shows the strains developed inside an element inside the crack trajectory; as expected, the three models coincide with the analytical solution: (a) the longitudinal strain grows linearly before bifurcation and faster and almost linearly after bifurcation, (b) the transverse strain grows linearly before bifurcation due to Poisson's effect and decreases afterwards due to elastic unloading and (c) the shear strain remains always null. The bottom curve shows the corresponding stresses: the longitudinal stress grows linearly before bifurcation and decreases exponentially after cracking, while the transverse and shear stresses remain null.

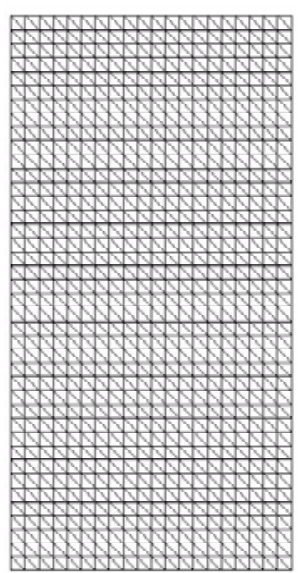

(a.1)

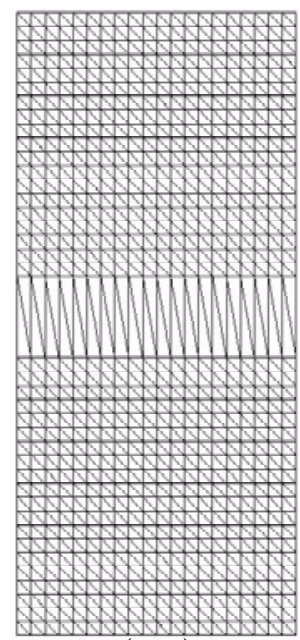

(a.2)

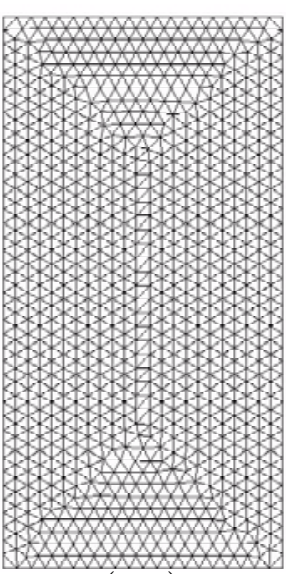

(b.1)

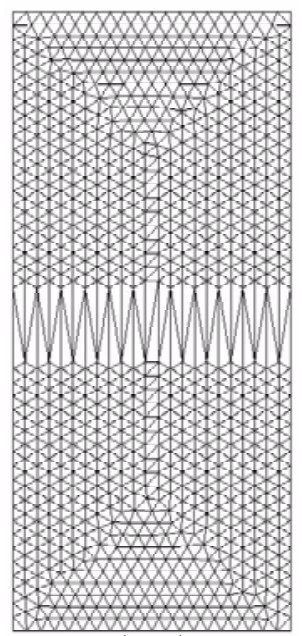

(b.2)

Figure 7: Meshes A and B and deformed shapes for the rectangular strip under tension 

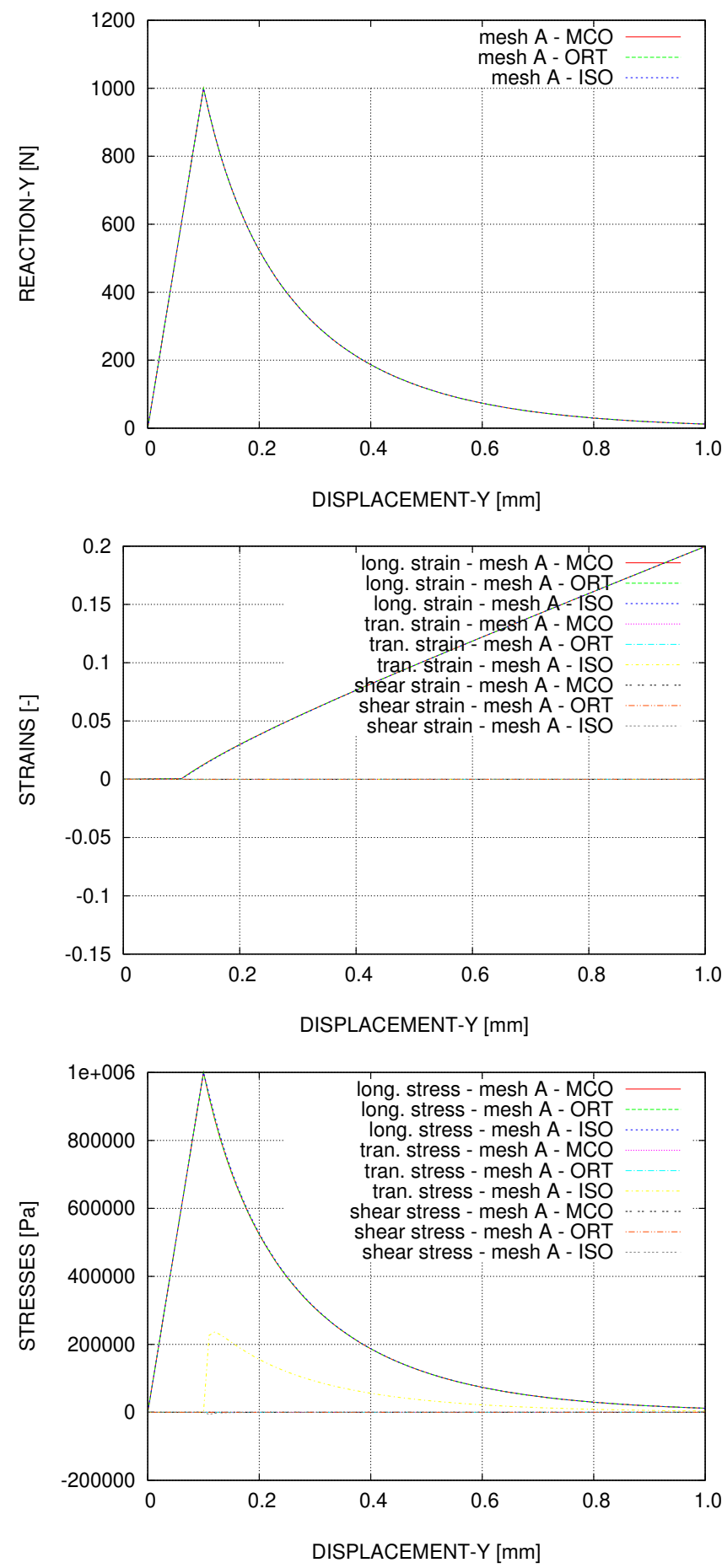

Figure 8: Results for rectangular strip using mesh A 


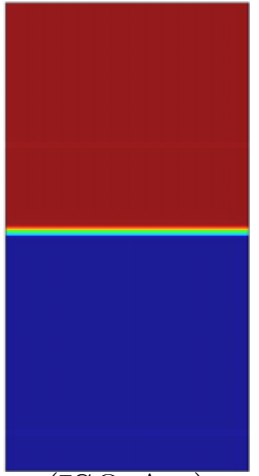

(ISO-A.a)

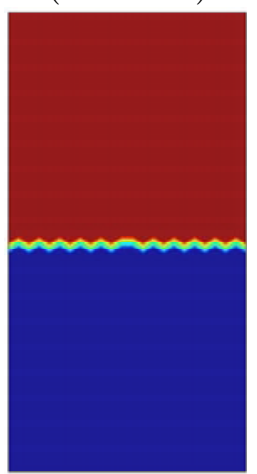

(ISO-B.a)

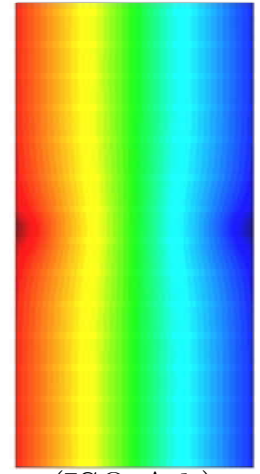

(ISO-A.b)

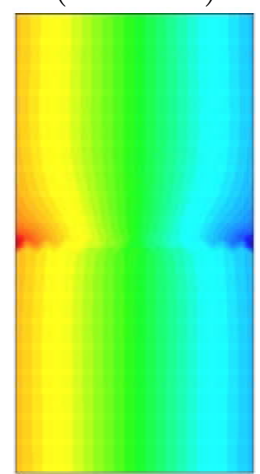

(ISO-B.b)

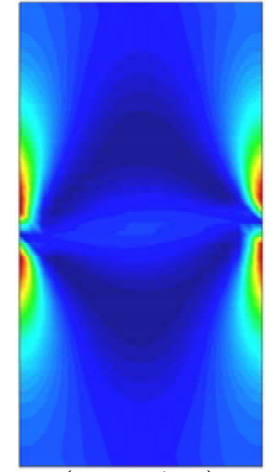

(ISO-A.c)

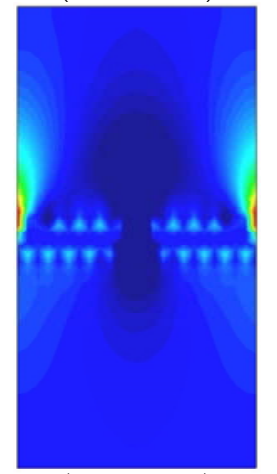

(ISO-B.c)

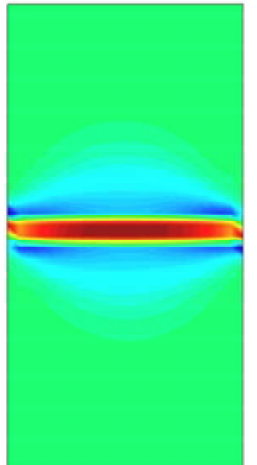

(ISO-A.d)

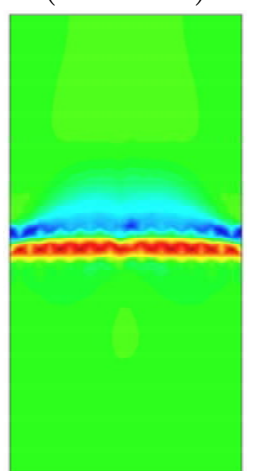

(ISO-B.d)

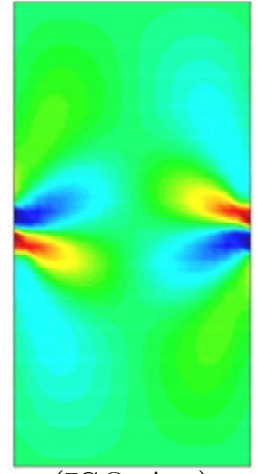

(ISO-A.e)

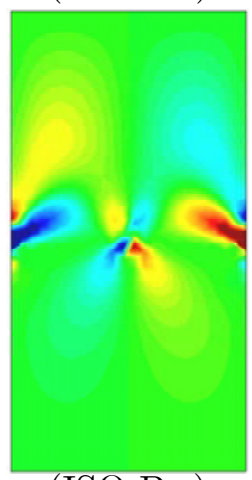

(ISO-B.e)

Figure 9: Results for rectangular strip using the isotropic model (top: mesh A, bottom: mesh B). Contours of: (a) vertical displacement, (b) horizontal displacement (c) vertical normal stress, (d) horizontal normal stress and (e) shear stress

In fact, the performance of both the associative and mesh corrected orthotropic models is identical and exact as, for this mesh, the crack is parallel to one of the element sides. The behaviour of the isotropic damage model is almost perfect, apart from one subtle aspect: it is unable to reproduce correctly Poisson's effect after cracking. To visualize this, Figure 9 (top) shows different contours for this analysis when the (half)-imposed vertical displacement is $\delta=0.05 \mathrm{~mm}$. Figure 9.ISO-A.a shows contours for the vertical displacement; all variation is contained inside the localization band. Figure 9.ISO-A.b shows contours for the horizontal displacement; here, a small amount of "necking" can be observed. It is due to the fact that, on one hand, the isotropic model preserves the value of the Poisson's ratio, while on the other, the longitudinal strain is different inside the localization band than outside. The effect of this necking is clear in the contours of the longitudinal stress (Figs. ISO-A.c), the transverse stress (Fig. ISO-A.d) and the shear stress (Fig. ISO-A.e). Particularly, Fig. ISO-A.d shows how the central band is under transverse tension as it is pulled out by the unloading elastic part of the strip. The values of the longitudinal stress are globally correct, but instead of being uniform they present oscillations of about $10 \%$ (Fig. ISO-A.c). 

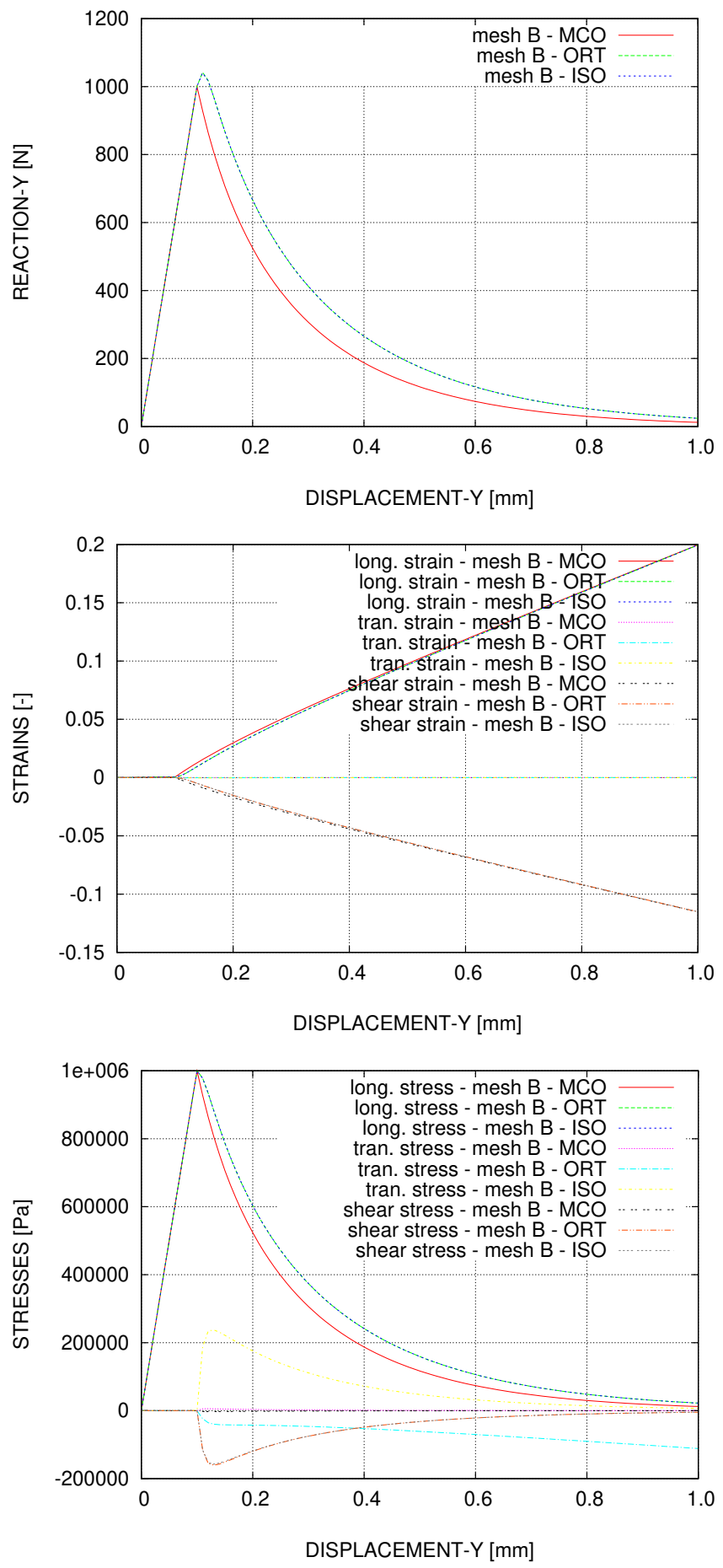

Figure 10: Results for rectangular strip using mesh B 


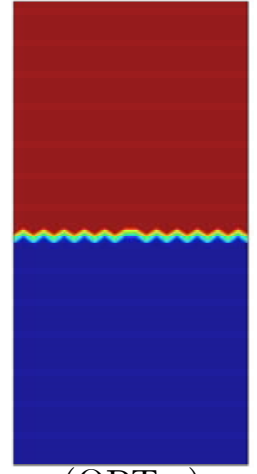

(ORT.a)

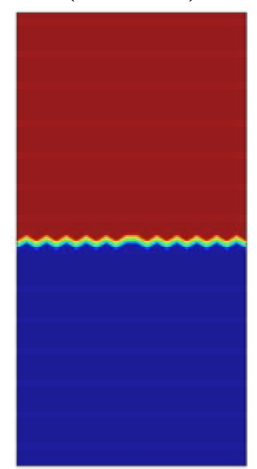

(MCO.a)

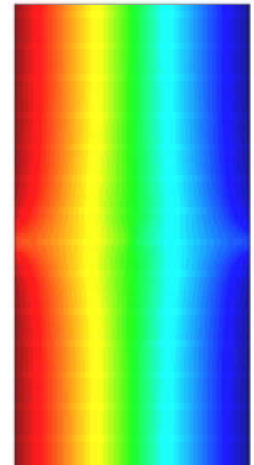

(ORT.b)

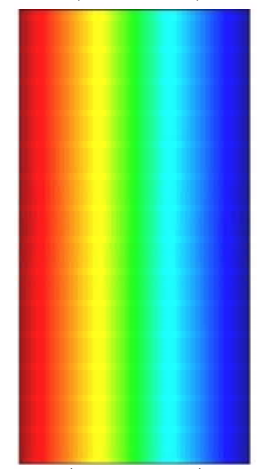

(MCO.b)

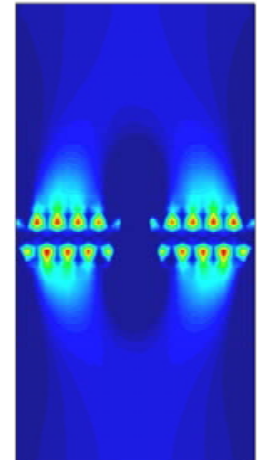

(ORT.c)

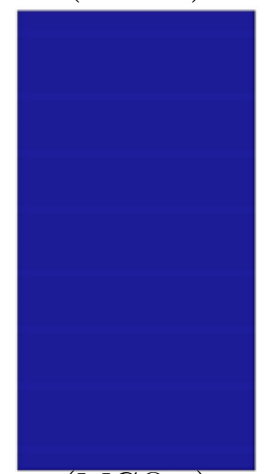

(MCO.c)

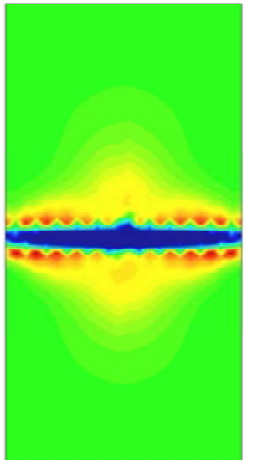

(ORT.d)

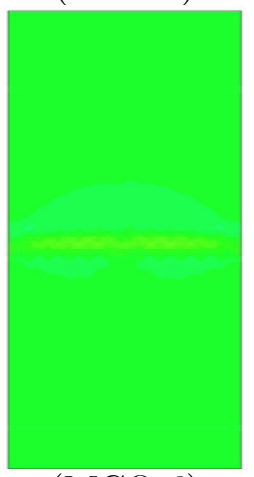

(MCO.d)

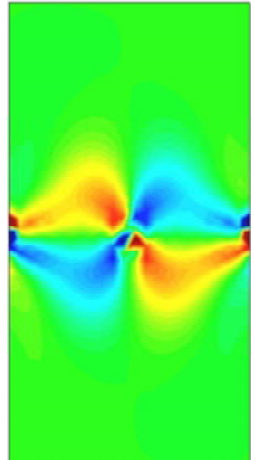

(ORT.e)

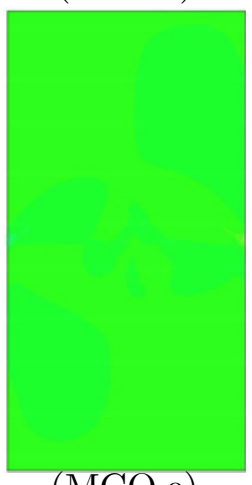

(MCO.e)

Figure 11: Results for rectangular strip using the orthotropic models in mesh B (top: associative, bottom: mesh corrected). Contours of: (a) vertical displacement, (b) horizontal displacement (c) vertical normal stress, (d) horizontal normal stress and (e) shear stress

The values of the transverse and shear stress, which should be exactly null, oscillate between $\pm 25 \%$ of the correct value of the longitudinal stress (Figs. ISO-A.d and ISO-A.e).

Figure 10 shows the results obtained with the same three constitutive models using miss-aligned mesh B. The top plot shows the load vs displacement curve, where the orthotropic mesh corrected model provides the exact solution, identical to the one obtained with mesh A, while the other two models give a slightly overestimated response, with coincident curves for both of them. The explanation for this is found in the other two plots below. The middle plot shows the strains developed inside an element inside the crack trajectory: (a) the longitudinal strain is very similar, although not identical, for the three models, and it behaves like in mesh A, (b) the transverse strain is also very similar (not identical) for the three models and to what happens in mesh A, but (c) the shear strain is not null and it grows linearly with the increasing longitudinal strain. This is the crucial point which relates to the miss-alignment of the mesh and causes the deficient response of the isotropic and associative orthotropic 
models. The bottom curve shows the corresponding stresses: (a) the longitudinal stress is only exactly correct for the MCO model, while the other two do not soften exactly as expected, (b) the transverse stress is only null in the MCO model, and not in the other two (for the ORT model it increases monotonically after cracking) and (c) the shear stress is only null for the MCO model, while reaching significant values in the other two.

Let us consider in detail the behaviour of the three models. Figure 9 (bottom) shows different contours for the analysis using the isotropic model and mesh B, when the (half)-imposed vertical displacement is $\delta=0.05 \mathrm{~mm}$. Figure 9.ISO-B.a shows contours for the vertical displacement; all variation is contained inside the zig-zagging localization band. Figure 9.ISO-B.b shows contours for the horizontal displacement; a small amount of "necking" can be observed, more pronounced in the top half of the strip than in the bottom half. The effect of this necking is also clear in the contours of the longitudinal stress (Figs. ISO-B.c), the transverse stress (Fig. ISO-B.d) and the shear stress (Fig. ISO-B.e). The values of the longitudinal stress are overestimated by $40 \%$ with respect to the correct uniform value, with oscillations of $35 \%$ (Fig. ISOB.c). The values of the transverse and shear stress, which should be null, oscillate between $\pm 30 \%$ of the value correct longitudinal stress (Figs. ISO-B.d and ISO-B.e).

Figure 11 shows the corresponding contours for the analyses using the orthotropic models and mesh $\mathrm{B}$, also when the (half)-imposed vertical displacement is $\delta=0.05$ $\mathrm{mm}$. The top contours correspond to the associative model (ORT) and the bottom pictures to the mesh corrected model (MCO). Figures 11.ORT.a and MCO.a show contours for the vertical displacement, both correct and identical to the results obtained with the isotropic model; all variation is contained inside the zig-zagging localization band. Figure 11.ORT.b shows contours for the horizontal displacement; here, a small amount of transverse "stretching" can be observed in the localization band, spurious and completely dependent on the orientation of the mesh. The effect of this stretching is also clear in the contours of the longitudinal stress (Fig. ORT.c), the transverse stress (Fig. ORT.d) and the shear stress (Fig. ORT.e). The values of the longitudinal stress are overestimated by $40 \%$ with respect to the correct uniform value, with oscillations of $10 \%$ (Fig. ORT.c). The values of the transverse and shear stress, which should be null, oscillate between $\pm 15 \%$ of the value correct longitudinal stress (Figs. ORT.d and ORT.e). On the other hand, Figure 11.MCO.b shows exact contours for the horizontal displacement obtained with the mesh corrected model. The corresponding contours for the longitudinal stress (Fig. MCO.c), the transverse stress (Fig. MCO.d) and the shear stress (Fig. MCO.e) are also correct, almost completely uniform, the only deviations from the analytical values being due to the tolerance used in the non-linear solution procedure.

\subsection{Notched slab}

The second example is a plane-stress notched slab subjected to mode I stretching. Figure 12 depicts the geometry of the problem; dimensions of the slab are $300 \times$ 
$360 \mathrm{~mm} \times \mathrm{mm}$ (width $\times$ height), the length and width of the notch are $167 \mathrm{~mm}$ and $3 \mathrm{~mm}$, respectively, and the thickness of the slab is $10 \mathrm{~mm}$. The load is applied at two bottom (rigid) pins (at $86.5 \mathrm{~mm}$ from the center of the beam) by imposing horizontal displacements of opposite sign at the left and right pins. The following material properties are assumed for the slab: Young's modulus $E=2 \mathrm{GPa}$, Poisson's ratio $\nu=0.2$, tensile strength $\sigma_{o}=2 \mathrm{MPa}$ and mode I fracture energy $G_{f}=75 \mathrm{~J} / \mathrm{m}^{2}$. The pins are elastic with: Young's modulus $E=200 \mathrm{GPa}$, Poisson's ratio $\nu=0.3$.

This example is selected because it represents a typical situation of propagating mode I fracture, as the initial notch extends upwards along the vertical central axis of the slab. On one hand, symmetry conditions allow to determine the path of crack propagation. On the other hand, the strain and stress fields are complex before and after cracking; therefore, substantial redistribution of stress is expected as cracking progresses. Also, this test case highlights the shortcomings of the spatial discretization used. The example is used to assess the ability of the isotropic (ISO), associative orthotropic (ORT) and mesh corrected orthotropic (MCO) damage models to reproduce these complex conditions. Because of the previous reasons, we will use both the fixed and rotating versions of the orthotropic models, with the directions of orthotropy fixed at the onset of cracking or updated at each time step to be aligned with the directions of principal effective stress (and strain).

The computational domain is discretized in an unstructured mesh with average mesh size of $h_{e}=5 \mathrm{~mm}$ (4,983 nodes, 9,633 elements). The mesh is shown in Fig. 13a.

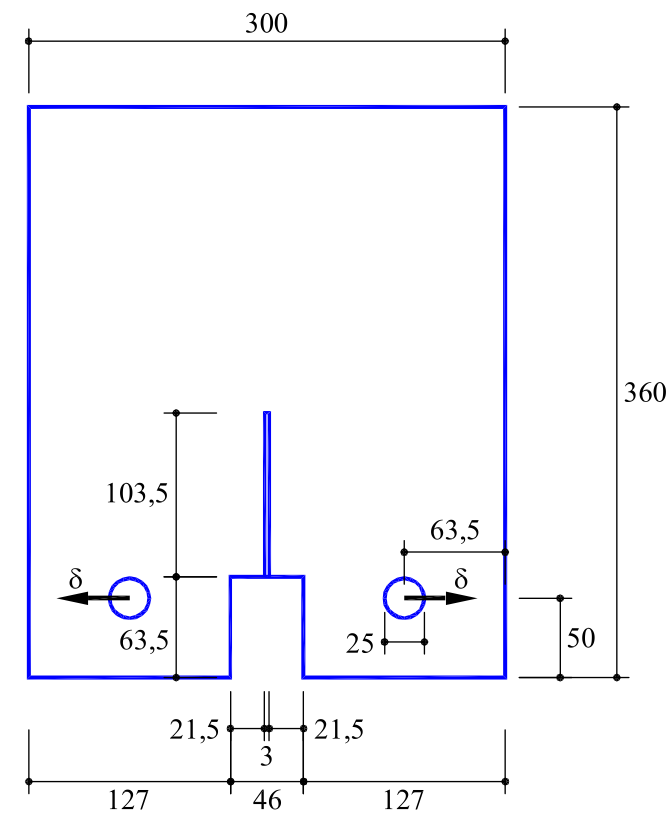

Figure 12: Geometry and loading of notched slab 


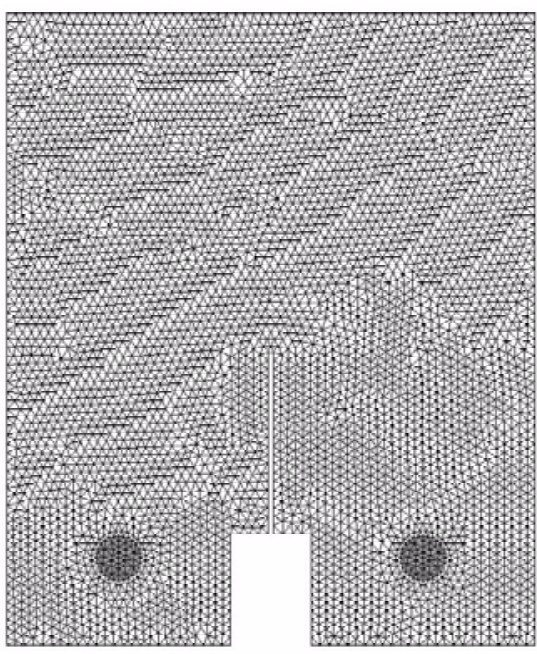

(a)

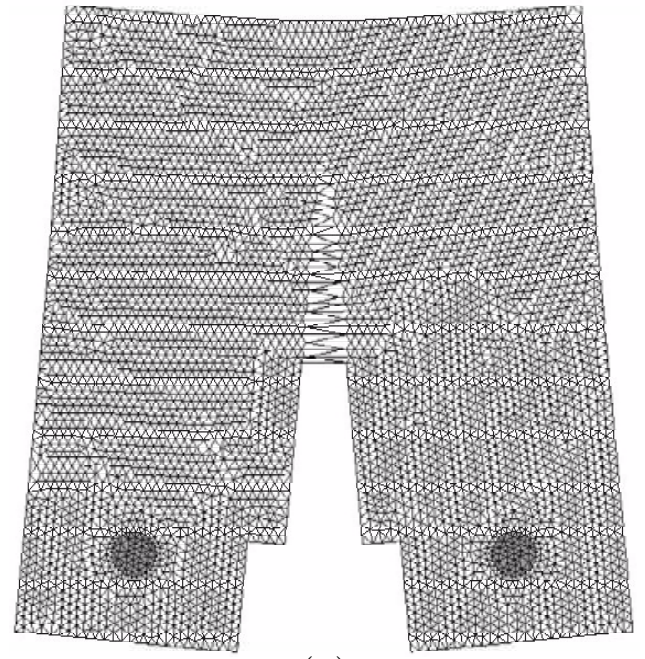

(b)

Figure 13: Mesh and deformed shape of notched slab

The computed deformed shape of the slab (using the mesh corrected model) is shown in Figure 13b (imposed horizontal displacement $\delta=0.4 \mathrm{~mm}$, with a displacement amplification factor of 50). As shown, at this stage the computed crack has progressed vertically about $125 \mathrm{~mm}$.

Figure 14 shows the results obtained with the three constitutive models. The top plot shows the load vs displacement curves, both for the fixed and rotating versions of the orthotropic models. It can be seen that the fixed orthotropic models lock almost completely, failing to show any trace of softening global response. This is the reason why fixed orthotropic models were abandoned in the past. On the other hand, the rotating mesh corrected orthotropic model displays the correct global behaviour: although non-linear behaviour starts very early, the global response shows positive stiffness until the crack is propagates about $10 \mathrm{~mm}$; after the peak load, global softening is observed. The isotropic model shows a similar response, but the peak load and the post-peak behaviour are over-estimated because of excessive dissipation. The standard rotating associative orthotropic model, as expected, fails to properly release the load after the peak; this is due to stress locking.

The middle plot illustrates the performance of the rotating mesh corrected orthotropic model, as it shows the stresses developed at the element located just at the tip of the initial notch, the first one to crack. Note how the horizontal normal stress, orthogonal to the crack, reaches the peak stress and immediately starts to soften. The vertical normal stress, parallel to the crack, continues to grow, and the shear stress starts to develop at the onset of cracking. For approximately $\delta=0.1 \mathrm{~mm}$, stress redistribution due to the cracking of the upper elements forces the release of the parallel stress and the reversal and eventual release of the shear stress. The sudden rotation 

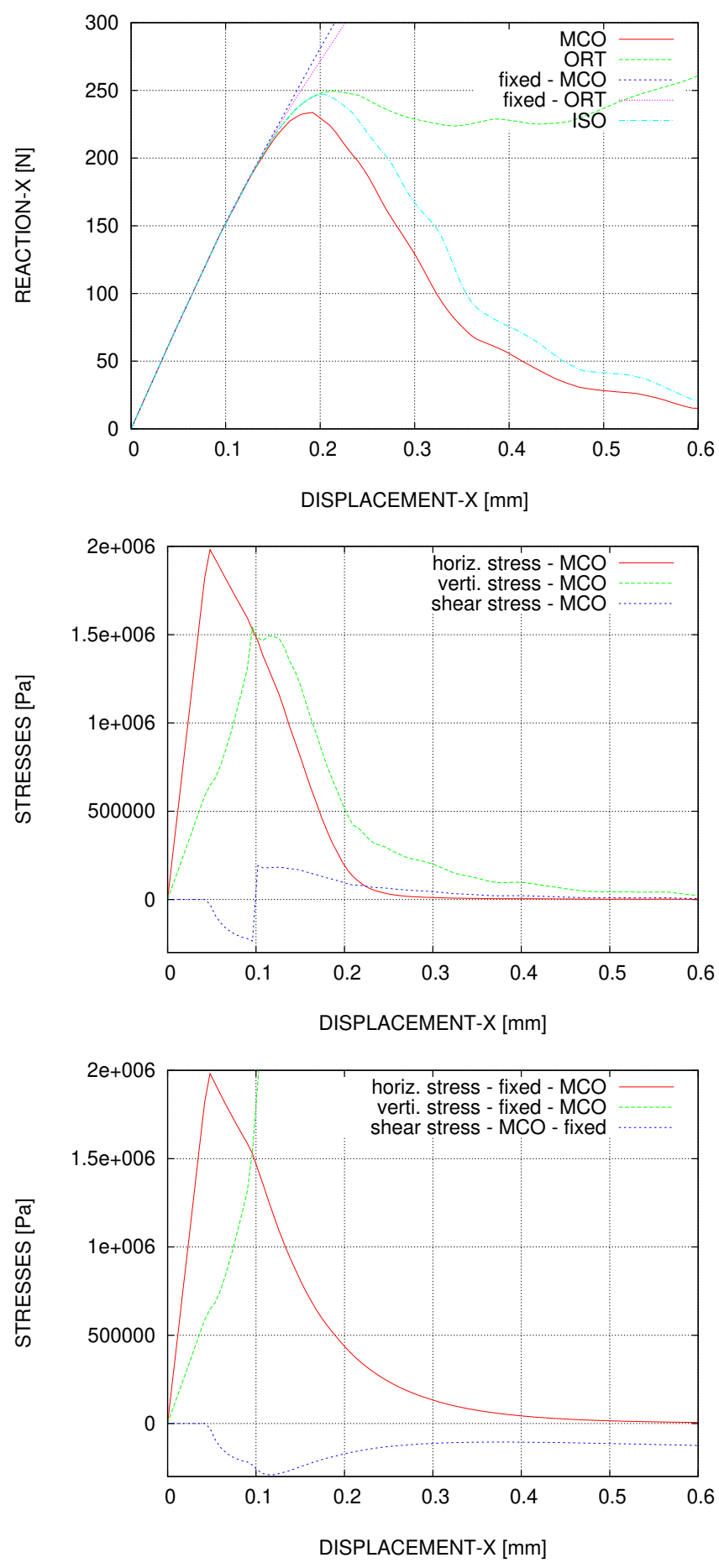

Figure 14: Results for notched slab 


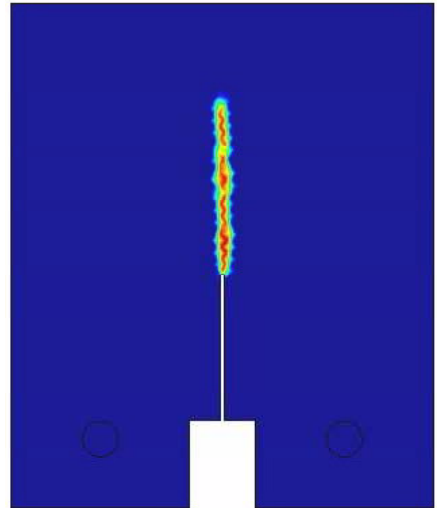

(a)

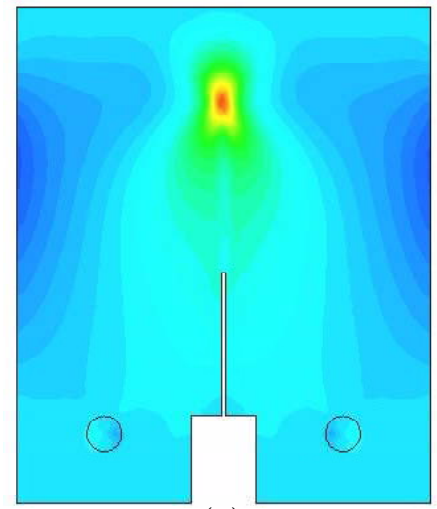

(c)

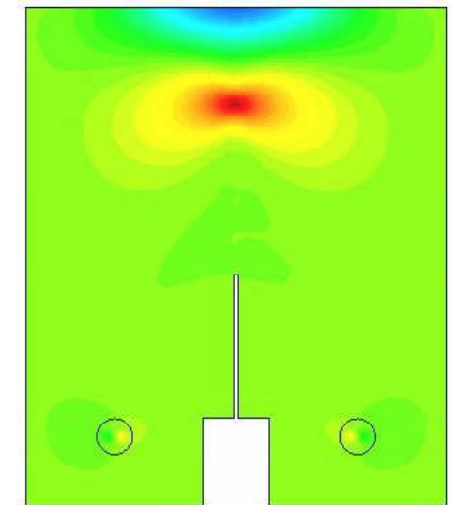

(b)

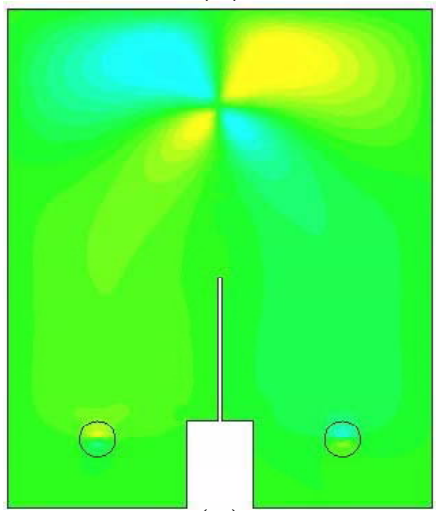

(d)

Figure 15: Results for the notched slab using the mesh corrected orthotropic model. Contours of: (a) damage index, (b) horizontal normal stress, (c) vertical normal stress and $(d)$ shear stress

of the principal strain directions in the element of attention is of about $50^{\circ}$ and this suffices to change the solitary node and, therefore, the structure of the inelastic strains from that point on. This accommodation of the inelastic strains is not possible if the axes of orthotropy are kept fixed. This is shown in the bottom plot, which shows corresponding results using the fixed mesh corrected orthotropic model. Note how in this case the parallel stress does not undergo any softening and how the shear stress does not reverse sign.

Let us now consider the global behaviour of three of the models. Figure 15 shows different contours for the analysis using the rotating mesh corrected orthotropic model, when the imposed horizontal displacement is $\delta=0.4 \mathrm{~mm}$. Figure 15.a shows contours for the damage index; all damage contained inside the zig-zagging vertical localization band. The crack has progressed upwards $125 \mathrm{~mm}$ from the notch tip. Figure 15.b shows contours for the horizontal normal stress; concentration of tensile stress marks the stress field at the tip of the propagating crack. This concentration is also clear in the contours of the vertical normal stress (Fig. 15c), and, faintly, in the shear stress 


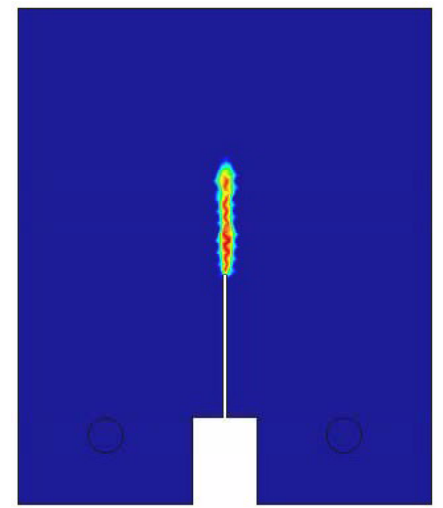

(a)

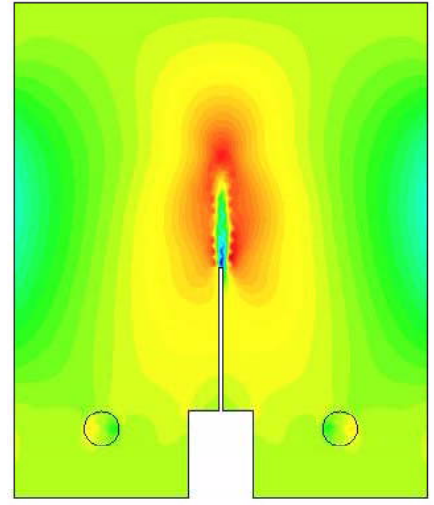

(c)

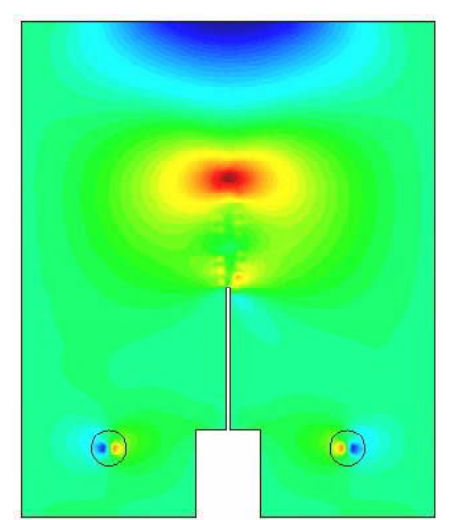

(b)

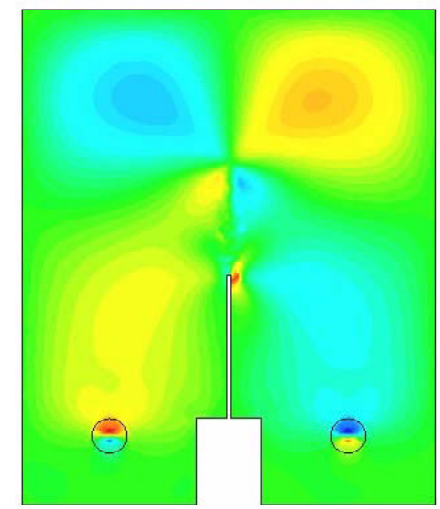

(d)

Figure 16: Results for the notched slab using the associative orthotropic model. Contours of: (a) damage index, (b) horizontal normal stress, (c) vertical normal stress and $(d)$ shear stress

(Fig. 15d). The overall behavior is as expected and no oscillations or stress locking is evident anywhere.

The situation is very different in Figure 16, where the corresponding contours for the analysis using the rotating associative orthotropic model are shown. Even if the imposed horizontal displacement is also $\delta=0.04 \mathrm{~mm}$, Figure 16.a shows that the crack has progressed insufficiently, about half as much as with the MCO model. Figures 16.b, 16.c and 16.d show that this is caused by substantial stress locking along the crack path.

Finally, Figure 17 shows contours for the isotropic model. Here, the crack has progressed upwards $110 \mathrm{~mm}$ (10\% less than with the MCO model). The reason can be found in Figures 17.b, 17.c and 17.d, which show that the stress locking caused by the spatial discretization is mitigated to a large extend, but not completely, by the isotropic nature of damage. 


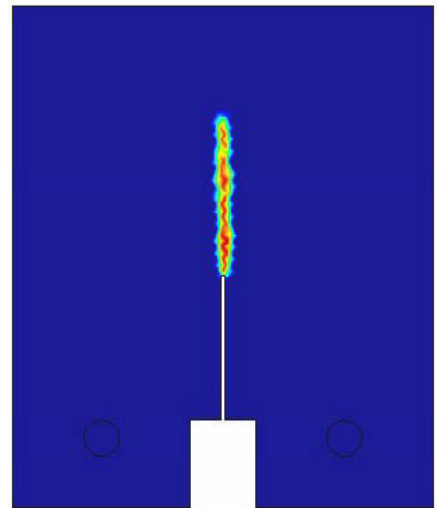

(a)

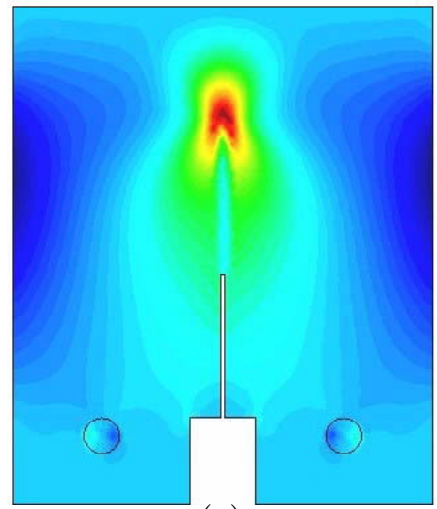

(c)

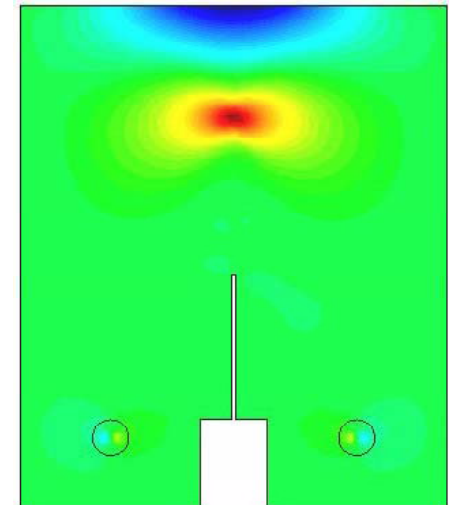

(b)

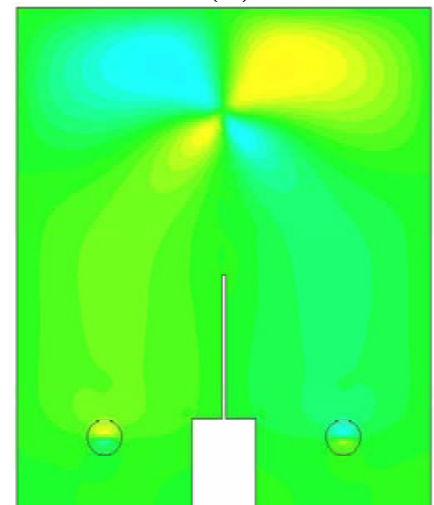

(d)

Figure 17: Results for the notched slab using the isotropic model. Contours of: (a) damage index, (b) horizontal normal stress, (c) vertical normal stress and (d) shear stress

\section{Conclusions}

This paper returns to the original spirit of the continuous crack approaches, where displacements jumps across the crack are smeared over the affected elements and the behaviour of the crack is established through a softening stress-(total)strain law, using standard finite elements, such as linear triangles, and local constitutive models, such as orthotropic continuum damage models. This option, proposed by Rashid in 1968 and still very popular among users of non linear FE codes, had been abandoned in academic circles due to observed stress locking effects and spurious mesh bias. Orthotropic models were first over-ruled by a step back to isotropic plasticity and damage models which do not show such evident locking effects. Later, the whole smeared approach was displaced in favor of discontinuous displacement approaches. Finally, even the local nature of the constitutive models has been questioned.

In this paper, the interest is focussed on the problem of locking in orthotropic constitutive models. First, a framework for non-associative orthotropic continuum 
damage models is provided. Next, a solution for the shear locking observed in the discrete problem is proposed in the form of a mesh corrected crack model where the structure of the inelastic strain tensor is linked to the geometry of the cracked element. The underlying idea is borrowed from the so-called strong discontinuity approach. The resulting formulation is easily implemented in standard non linear FE codes and is suitable for engineering applications in 2D and 3D.

Numerical examples show, on one hand, that the use of the proposed mesh corrected crack model notoriously avoids the dependence of the computed structural response on the mesh directional alignment; on the other, that relating the softening parameter of the constitutive model to the fracture energy of the material and to the size of the finite elements in the localization band enables to control the dissipated energy during the localization (fracture) process, yielding a correct structural response in the softening regime.

Finally, computed solutions indicate that continuous displacement interpolations can reproduce problems involving crack propagation as satisfactorily as the discontinuous approaches.

\section{Acknowledgments}

The invaluable help of Prof. R. Codina, expressed in the form of so many suggestions and fruitful discussions, is gratefully acknowledged.

\section{References}

[1] Belytschko, T. and Black, T. (1999). Elastic crack growth in finite elements with minimal remeshing. Comp. Meth. in Appl. Mech. and Eng., 45(5), 601-620.

[2] Möes, N., Dolbow, J. and Belytschko, T. (1999). A finite element method for crack growth without remeshing. Int. J. Num. Meths. in Engng., 46, 131-150.

[3] Sukumar, N., Möes, N., Moran, B. and Belytschko, T. (2000). Extended finite element method for three-dimensional crack modelling. Int. J. Num. Meths. in Engng., 48, 1549-1570.

[4] Simo, J.C., Oliver, J. and Armero, F. (1993). An analysis of strong discontinuities induced by strain-softening in rate-independent inelastic solids. Computational Mechanics, 12, 49-61.

[5] Oliver, J. (1995). Continuum modeling of strong discontinuities in solid mechanics using damage models. Computational Mechanics, 17, 277-296.

[6] Oliver, J., Cervera, M. and Manzoli, O. (1999). Strong discontinuities and continuum plasticity models: the strong discontinuity approach. Int. J. of Plasticity, 15, 319-351. 
[7] Jirásek, M. (2000) Comparative study on finite elements with embedded discontinuities. Comp. Meth. in Appl. Mech. and Eng., 188, 307-330.

[8] Jirásek, M. (2001) Embedded crack model: I. Formulation. Int. J. Num. Meths. in Engng., 50, 1269-1290.

[9] Jirásek, M. (2001) Embedded crack model: II: Combination with smeared cracks. Int. J. Num. Meths. in Engng., 50, 1291-1305.

[10] Mosler, J. and Meschke, G. (2004). Embedded crack vs. smeared crack models: a comparison of elementwise discontinuous crack path approaches with emphasis on mesh bias. Comp. Meth. in Appl. Mech. and Eng., 193, 3351-3375.

[11] Manzoli, O.L. and Shing, P.B. (2006). A general technique to embed non-uniform discontinuities into standard solid finite elements. Computers and Structures, 84, 742-757.

[12] Sancho, J.M., Planas, J., Cendón, D.A., Reyes, E. and Gálvez, J.C. (2007). An embedded cohesive crack model for finite element analysis of concrete fracture. Engineering Fracture Mechanics, 74(1-2), 75-86.

[13] Sancho, J.M., Planas, J., Fathy, A.M., Gálvez, J.C. and Cendón, D.A. (2007). Three-dimensional simulation of concrete fracture using embedded crack elements without enforcing crack path continuity. Int. J. for Num. and Anal. Meth. in Geomechanics, in press.

[14] Rashid, Y. (1968). Analysis of prestrssed concrete pressure vessels. Nuclear Engineering and Design, 7, 334-344.

[15] Clough, R.W. (1962). The stress distribution of Norfolk Dam. Structures and Materials Research, Department of Civil Engineering Series 100, 19, University of California, Berkeley, California, USA.

[16] Ngo, D. and Scordelis, A.C. (1967). Finite element analysis of reinforced concrete beams. ACI Journal, 64(14), 152-163.

[17] Nilson, A.H. (1968). Nonlinear Analysis of Reinforced Concrete by the Finite Element Method. ACI Journal, 65(9), 757-776.

[18] Jirásek, M. and Zimmermann, T. (1998). Analysis of Rotating Crack Model. Journal of Engineering Mechanics (ASCE), 124(8), 842-284.

[19] Cervera, M. and Chiumenti, M. (2006). Smeared crack approach: back to the original crack. Int. J. for Num. and Anal. Meth. in Geomechanics, 30, 1173-1199.

[20] Cervera, M. and Chiumenti, M. (2006). Mesh objective tensile cracking via a local continuum damage model and a crack tracking technique. Comp. Meth. in Appl. Mech. and Eng., 196(1-3), 304-320. 
[21] Cervera, M., Chiumenti, M. and Agelet de Saracibar, C. 2003. Softening, localization and stabilization: capture of discontinuous solutions in J2 plasticity. Int. J. for Num. and Anal. Meth. in Geomechanics, 28, 373-393.

[22] Cervera, M., Chiumenti, M. and Agelet de Saracibar, C. 2003. Shear band localization via local $\mathrm{J}_{2}$ continuum damage mechanics. Comp. Meth. in Appl. Mech. and Eng., 193, 849-880.

[23] Cervera, M., Oliver, J., and Faria, R. (1995). Seismic evaluation of concrete dams via continuum damage models. Earth. Engng. Struc. Dyn., 24, 1225-1245.

[24] Cervera, M., Oliver, J., and Manzoli, O. (1996). A rate-dependent isotropic damage model for the seismic evaluation of concrete dams. Earth. Engng. Struc. Dyn., $25,987-1010$.

[25] Faria, R., Oliver, J., and Cervera, M. (1998). A strain-based plastic viscousdamage model for massive concrete structures. Int. J. Solids and Structures, $35(14): 1533-1558$.

[26] Faria, R., Oliver, J., and Cervera, M. (2004). Modeling Material Failure in Concrete Structures under Cyclic Actions. J. of Structural Engineering, ASCE, 130, 1997-2005.

[27] Simó, J. C. and Ju, J. W. (1987). Strain- and stress-based continuum damage models - I. formulation. Int. J. Solids and Structures, 23, 821-840.

[28] de Borst, R. (2002). Fracture in quasi-brittle materials: a review of continuum damage-based approaches. Engineering Fracture Mechanics, 69, 95-112.

[29] Lemaitre, J. and Chaboche, J. L. (1978). Aspects phénoménologiques de la rupture par endommagement. J. Méc. Appl., 2, 317-365.

[30] Cervera, M., Chiumenti and Agelet de Saracibar, C. (2002). COMET: COupled MEchanical and Thermal analysis. Data Input Manual, Version 5.0, Technical report IT-308, www.cimne.upc.es.

[31] GiD: the personal pre and post-processor (2002). www.gid.cimne.upc.es. 